\title{
Simulating synchrotron radiation in accelerators including diffuse and specular reflections
}

\author{
G. Dugan and D. Sagan \\ Cornell University, Ithaca, New York 14850, USA
}

(Received 23 November 2016; published 24 February 2017)

\begin{abstract}
An accurate calculation of the synchrotron radiation flux within the vacuum chamber of an accelerator is needed for a number of applications. These include simulations of electron cloud effects and the design of radiation masking systems. To properly simulate the synchrotron radiation, it is important to include the scattering of the radiation at the vacuum chamber walls. To this end, a program called Synrad3D has been developed which simulates the production and propagation of synchrotron radiation using a collection of photons. Photons generated by a charged particle beam are tracked from birth until they strike the vacuum chamber wall where the photon is either absorbed or scattered. Both specular and diffuse scattering is simulated. If a photon is scattered, it is further tracked through multiple encounters with the wall until it is finally absorbed. This paper describes the Synrad3D program, with a focus on the details of its scattering model, and presents some examples of the program's use.
\end{abstract}

DOI: $10.1103 /$ PhysRevAccelBeams.20.020708

\section{INTRODUCTION}

Calculation of the synchrotron radiation flux within an accelerator vacuum chamber is important for a number of applications. For example, the electron cloud effect [1-6] has been a major performance-limiting issue for many accelerators in the past, and will need to be mitigated in future accelerators. In lepton accelerators and very-highenergy hadron accelerators, the cloud is seeded by photoelectrons, which are generated by synchrotron radiation. Understanding the evolution of the electron cloud and its effect on the beam requires a detailed understanding of where the photoelectrons are created. This is true since electrons will tend to follow the local magnetic field lines. Thus an electron produced at the top or bottom of the vacuum chamber in a bend with a vertical magnetic field will behave quite differently than an electron produced horizontally to the side. Since photoelectrons are created where photons of the requisite energy are absorbed, the photon absorption distribution has a profound effect on the electron cloud.

Simulation of synchrotron radiation is also needed in the design of radiation masking systems [7] to protect sensitive areas of the machine from the radiation flux. Additionally, vacuum pumping of exposed chamber surfaces is affected by the distribution of synchrotron radiation.

To properly simulate the effects of synchrotron radiation, it is important to include the scattering of the radiation at the

Published by the American Physical Society under the terms of the Creative Commons Attribution 4.0 International license. Further distribution of this work must maintain attribution to the author(s) and the published article's title, journal citation, and DOI. vacuum chamber walls. Areas that do not have any direct radiation shining on them may still see a significant flux due to scattering. To this end, a program called Synrad3D [8] has been developed which simulates synchrotron radiation by tracking photons generated by a charged particle beam from birth through scattering - both specular and diffuse-in multiple wall encounters, to eventual absorption.

In Sec. II and Sec. III below, this paper describes the Synrad3D program and its scattering model. In Sec. IV, the results of benchmarking the model against data are presented. Several examples of Synrad3D photon production, transport and absorption simulations, in which diffuse scattering is included in the simulation, are given in Sec. V. After the summary in Sec. VI, a detailed discussion of the derivation of the scattering model equations, and how they are used in Synrad3D, is presented in the Appendix.

\section{SYNRAD3D}

As part of the research for the CesrTA test accelerator [9], the Synrad3D $[8,10]$ program has been developed to track synchrotron radiation photons generated in storage rings and linacs. Synrad3D is based upon the Bmad software library [11]. Bmad is a toolkit for the simulation of charged particle as well as X-ray beams. Associated with Bmad are a number of programs used for lattice design and analysis, intrabeam scattering calculations, spin tracking, etc. Synrad3D, as well as Bmad, is open source and can be downloaded from the web as part of the Bmad distribution package [12].

The motivation for developing Synrad3D was to estimate the energy and position distribution of photons absorbed on the vacuum chamber walls, which are critical inputs to codes which model the growth of electron clouds. Synrad3D includes both specular and diffuse scattering from the walls. For the scattering calculation, Synrad3D takes as input 
the specular photon reflection probability as a function of angle and photon energy for a smooth surface. Using this, along with input values for the rms surface roughness and transverse correlation length, Synrad3D calculates the diffuse and specular scattering probabilities for the rough surface using an analytical model derived from Beckmann [13,14].

The smooth-surface photon reflectivity which Synrad3D uses as the basis for the scattering calculation is dependent upon the material properties of the surface. Different smooth-surface reflectivities can be assigned to different parts of the vacuum chamber. If not specified by the user, the default smooth-surface reflectivity model used is that for an aluminum chamber with a $10 \mathrm{~nm}$ carbon film on the surface. This smooth-surface reflectivity model is illustrated in Fig. 1. The data for this model was obtained from a Lawrence Berkeley National Laboratory (LBNL) x-ray scattering database [15].

Synrad3D can handle machines where the geometry is not planar and can handle intersecting beam lines. For example, Synrad3D can handle the geometry created by $\mathrm{x}$-ray beam lines connected to a storage ring. Additionally, Synrad3D can handle a wide variety of vacuum chamber profiles.

The vacuum chamber wall is defined in Synrad3D by the union of a number of "subchambers" as shown in Fig. 2. A photon is considered within the vacuum chamber if, and only if, it is inside at least one of the subchambers. Each subchamber is defined by a number of cross sections, as shown in Fig. 3, and each cross section is defined by a number of vertices. A straight line or the arc of an ellipse can be used to connect the vertices. The one restriction on

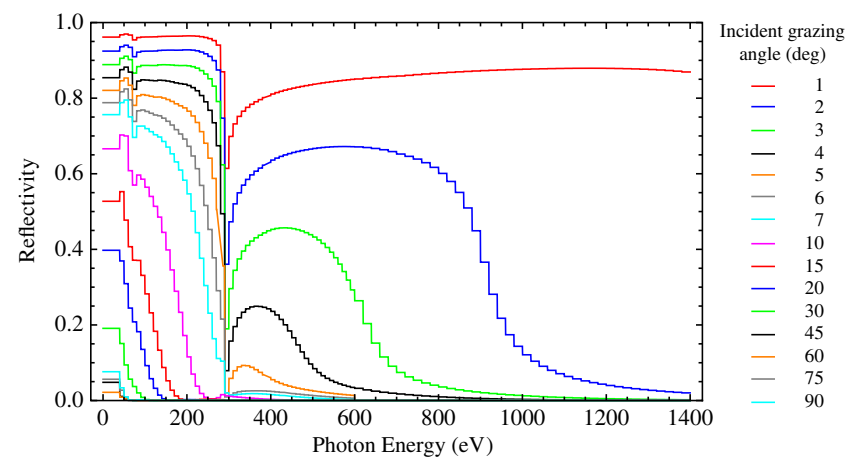

FIG. 1. Smooth surface photon reflectivity $R$ for a $10 \mathrm{~nm} \mathrm{C} \mathrm{film}$ on Al substrate [15].

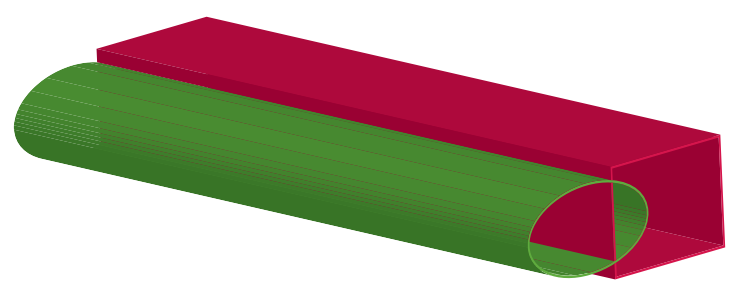

FIG. 2. The vacuum chamber is the union of a number of subchambers. This figure illustrates this showing two subchambers-one colored green and the other colored red.
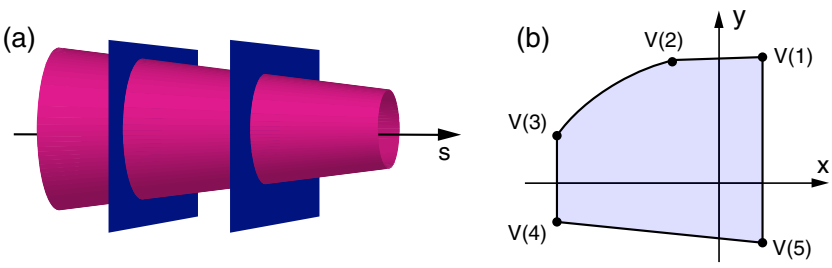

FIG. 3. A subchamber is defined by a number of cross-sectional slices. (A) A subchamber (red) and two cross-sectional slices (blue). (B) A given cross section is defined by a number of vertices.

subchamber construction is that the volume between adjacent cross sections should be convex. By imposing this restriction, Synrad3D can efficiently track photons.

Synrad3D uses Monte Carlo techniques to generate simulated photons based on the standard synchrotron radiation formulas for charged beam particles traversing dipoles, quadrupoles and wigglers, in the lattice of an accelerator.

Photons are generated with respect to the particle beam's actual orbit (which may differ from the zero orbit), so the effect of variations in the orbit can be studied. The particle beam size is also taken into account when generating the photon starting positions. The emittance needed to calculate the beam size can be supplied by the user or is calculated from the standard synchrotron radiation formulas.

\section{SCATTERING MODEL}

Generally, the probability of specular reflection of a photon from a rough surface depends on the rms surface roughness $\sigma$, the photon wavelength $\lambda$, the incident and scattering angles, and the atomic properties of the surface. An explicit formula for this probability is (see Beckmann [13], or Eq. (A78) in the Appendix)

$$
P_{\text {spec }}=R \mathrm{e}^{-g},
$$

in which

$$
g=\frac{4 \pi^{2} \sigma^{2}(x+y)^{2}}{\lambda^{2}}
$$

$x$ is the cosine of the incident polar angle, and $y$ is the cosine of the scattered polar angle. The atomic properties of the surface are reflected in the smooth-surface photon reflectivity $R$.

For a typical technical vacuum chamber surface, the rms surface roughness $\sigma \sim 200 \mathrm{~nm}$ is greater than most of the $\mathrm{x}$-ray wavelengths of interest. In this regime, except at very small grazing angles, diffuse scattering from the surface dominates over specular reflection. This is illustrated in Fig. 4, which is based on Eq. (1), but with $R=1$.

The theory of diffuse scattering of electromagnetic waves from random rough surfaces is a well-developed subject, and is covered in detail in Beckmann [13] and Ogilvy [14]. The approach is based on Kirchhoff (scalar) diffraction theory; this approach has been used successfully 


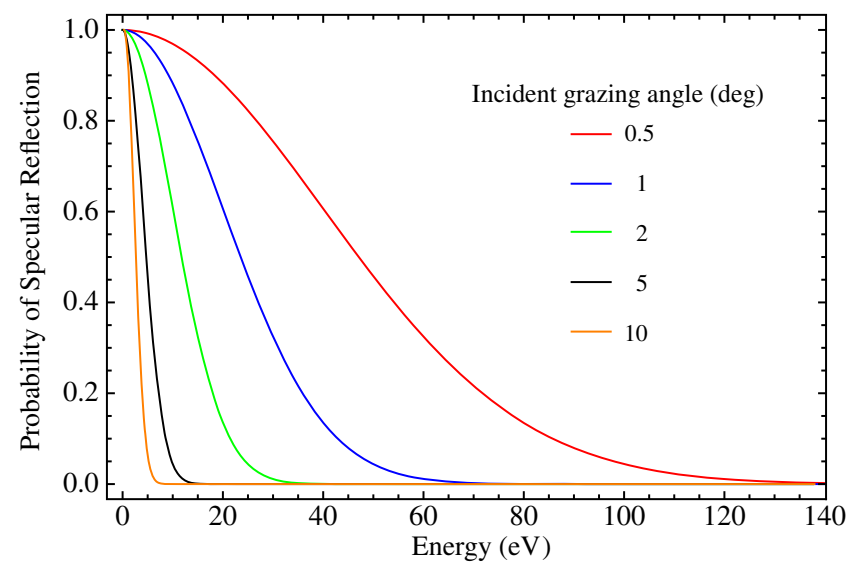

FIG. 4. Specular reflection probability [13], vs. photon energy and angle, for an rms surface roughness of $\sigma=200 \mathrm{~nm}$. The curves have been calculated from Eq. (1) with $R=1$.

to describe the scattering of soft $\mathrm{x}$-rays from metal surfaces $[16,17]$. In Synrad3D, we assume a Gaussian form for both the surface height distribution ( $\mathrm{rms} \sigma$ ) and for the transverse autocorrelation (the same in both transverse directions, with autocorrelation coefficient $T$ ).

The most general expression for the diffusely scattered power involves an infinite sum [see Eq. (A97)]. This full expression is used in Synrad3D. However, the expression simplifies substantially in the limit $g \gg 1$. This condition is satisfied (except for very small grazing angles) for rough surfaces, corresponding to technical vacuum chambers, and for high energy photons, for which typically $\sigma \gg \lambda$. In this limit, the average diffusely scattered power per unit solid angle is given by [see Eq. (A111)]

$$
\left\langle\frac{d P_{2}}{d \Omega_{2}}\right\rangle=P_{0} \frac{R \tau^{2}}{4 \pi y} \frac{(1+x y-h \zeta)^{2}}{(x+y)^{4}} \mathrm{e}^{-\frac{\tau^{2}\left(2-x^{2}-y^{2}-2 h \zeta\right)}{4(x+y)^{2}}},
$$

in which

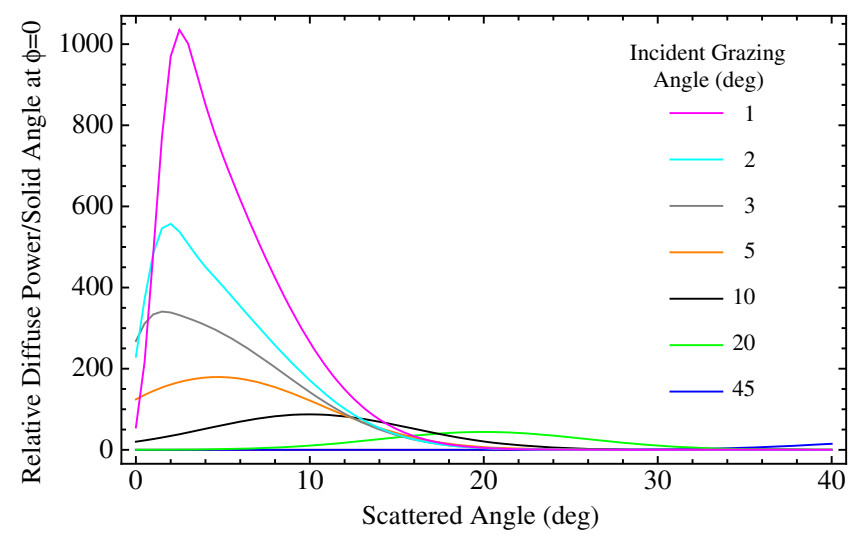

FIG. 5. Diffuse scattering polar angular distributions for $30 \mathrm{eV}$ photons, $\sigma=200 \mathrm{~nm}$ and $T=5500 \mathrm{~nm}$. The full diffuse scattering expression [Eq. (A97)] has been used to calculate these curves.

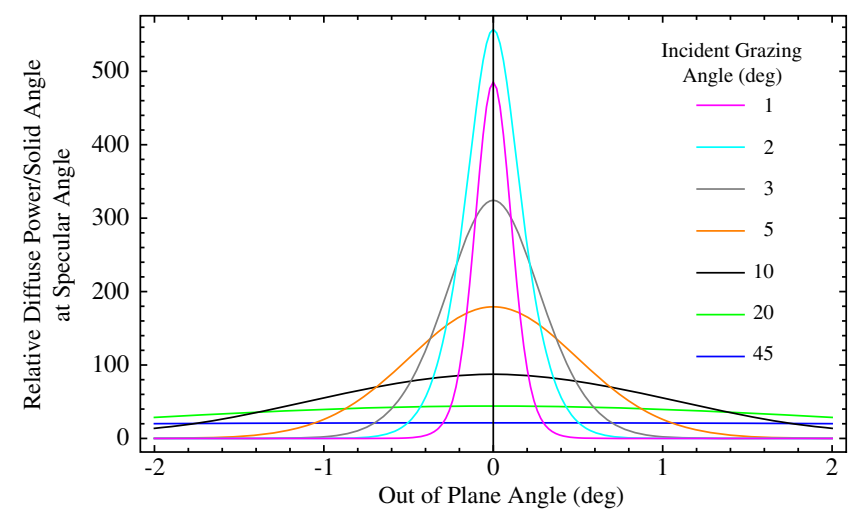

FIG. 6. Diffuse scattering out-of-plane angular distributions for $30 \mathrm{eV}$ photons, $\sigma=200 \mathrm{~nm}$ and $T=5500 \mathrm{~nm}$. The full diffuse scattering expression [Eq. (A97)] has been used to calculate these curves.

$$
\begin{gathered}
h=\sqrt{1-x^{2}} \sqrt{1-y^{2}} \\
\zeta=\cos \phi .
\end{gathered}
$$

In this expression, $P_{0}$ is the incident power, $R$ is the smooth-surface photon reflectivity, and $\phi$ is the scattering angle out of the plane of incidence. Note that the scattered power depends on the ratio $\tau=T / \sigma$, and not on $T$ or $\sigma$ separately, and, in the $g \gg 1$ limit, the distribution is independent of photon wavelength.

The smooth-surface photon reflectivity $R$ depends on the atomic structure of the surface materials (including any thin layers which may be deposited on the surface). The surface roughness parameters $\sigma$ and $T$ depend on the geometry of the surface deviations from a perfect plane. These parameters may be determined from inspection of the vacuum chamber surface, for example, using an atomic force microscope.

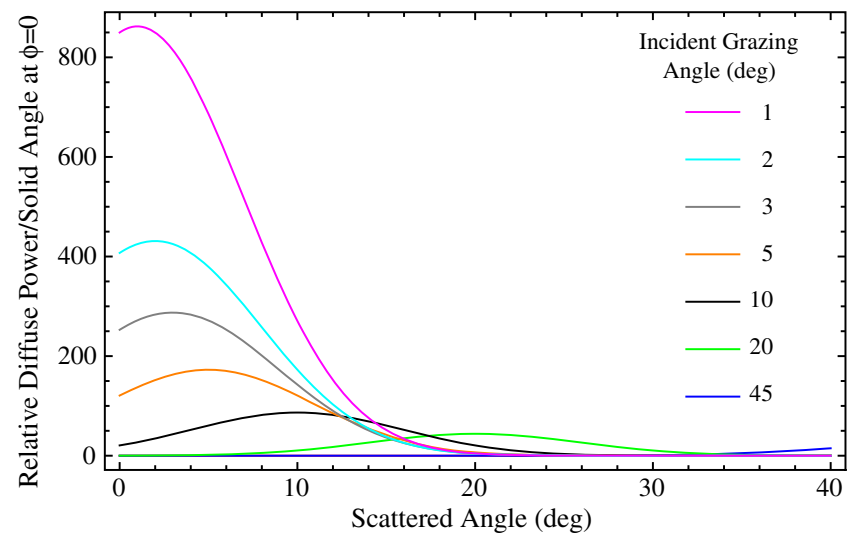

FIG. 7. Diffuse scattering polar angular distributions for high energy photons, $\sigma=200 \mathrm{~nm}$ and $T=5500 \mathrm{~nm}$. The curves are calculated from the approximate relation given in Eq. (3). In this case, high-energy photons correspond to $\lambda \ll \sigma$, i.e., $E \gg 6 \mathrm{eV}$. 


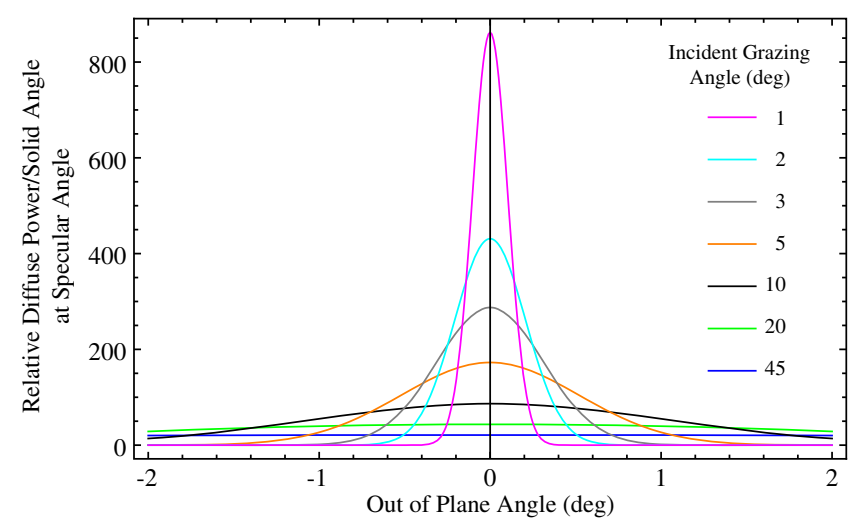

FIG. 8. Diffuse scattering out-of-plane angular distributions for high energy photons, $\sigma=200 \mathrm{~nm}$ and $T=5500 \mathrm{~nm}$. The curves are calculated from the approximate relation given in Eq. (3). In this case, high-energy photons correspond to $\lambda \ll \sigma$, i.e., $E \gg 6 \mathrm{eV}$.

For $\sigma=200 \mathrm{~nm}$ and $T=5500 \mathrm{~nm}$, which are the default values used by Synrad3D, diffuse scattering distributions for $30 \mathrm{eV}$ photons are shown in Fig. 5 and Fig. 6. At this low photon energy, the approximation $g \gg 1$ does not hold in general, and the full diffuse scattering formalism [Eq. (A97)] is used to compute these distributions. Diffuse scattering distributions for high energy photons, for which $g \gg 1$ are shown in Fig. 7 and Fig. 8. These distributions have been computed from Eq. (3).

\section{BENCHMARKING}

\section{A. DA $\Phi$ NE benchmarking}

To benchmark the scattering model for a typical vacuum chamber surface, we have relied on measurements [18] of $\mathrm{x}$-ray scattering from an aluminum vacuum chamber surface made at $D A \Phi N E$. For these measurements, the rms surface roughness of the sample was reported to be $200 \mathrm{~nm}$.

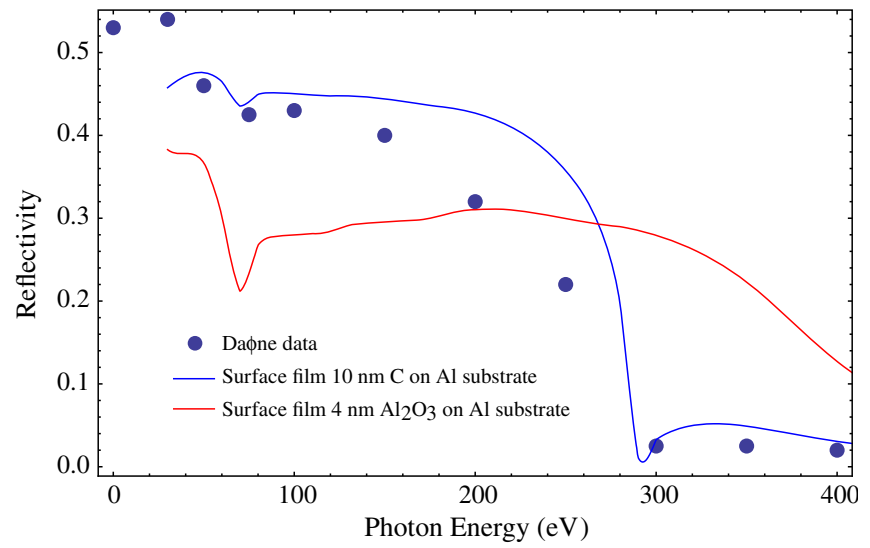

FIG. 9. Comparison of data [18] and model for diffuse scattering at $5^{\circ}$ from a rough $(\sigma=200 \mathrm{~nm})$ surface layer on an aluminum substrate.

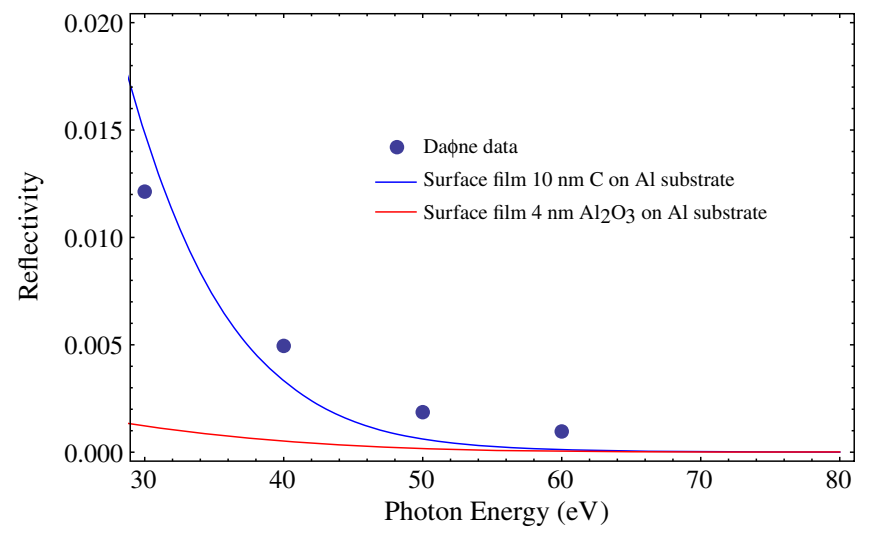

FIG. 10. Comparison of data [18] and model for diffuse scattering at $45^{\circ}$ from a rough $(\sigma=200 \mathrm{~nm})$ surface layer on an aluminum substrate.

The theory of diffuse scattering discussed above has been used, together with smooth surface reflectivity results taken from an x-ray database [15], to predict the scattering and compare with the measurements. From these comparisons, the best-fit value for the transverse autocorrelation parameter, $T$, was found to be $5500 \mathrm{~nm}$. As discussed by Dugan and Sagan [19], it was found that the smoothsurface reflectivity corresponding to a $10 \mathrm{~nm}$ carbon film on an aluminum substrate was needed to fit the data [The presence of a carbon (or carbon monoxide) film on technical vacuum chamber surfaces is a well-known phenomenon]. The assumption of an aluminum oxide surface film was not consistent with the data. The data and the corresponding fits are shown in Fig. 9,10, and 11.

\section{B. Benchmarking with other x-ray scattering measurements}

Additional comparisons of this theory with measurements have been reported in [20]. In that paper, the general outline of the theory is given, but the paper lacks a detailed

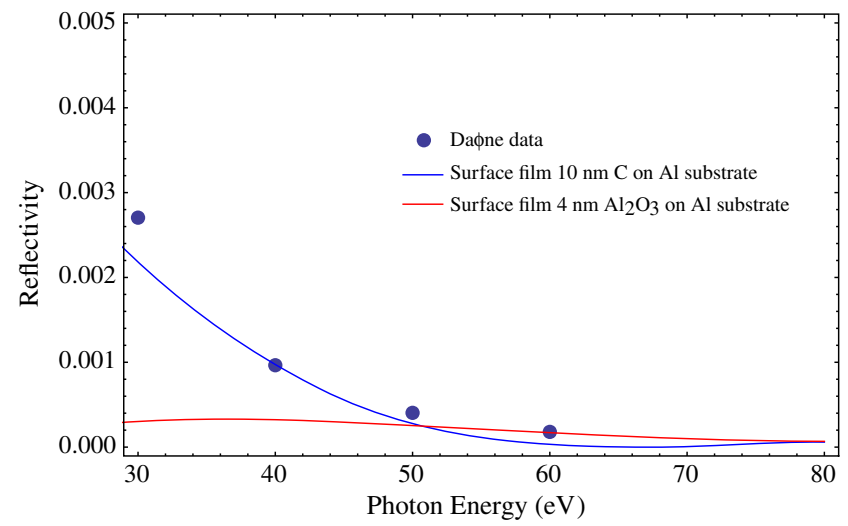

FIG. 11. Comparison of data [18] and model for diffuse scattering at $85^{\circ}$ from a rough $(\sigma=200 \mathrm{~nm})$ surface layer on an aluminum substrate. 


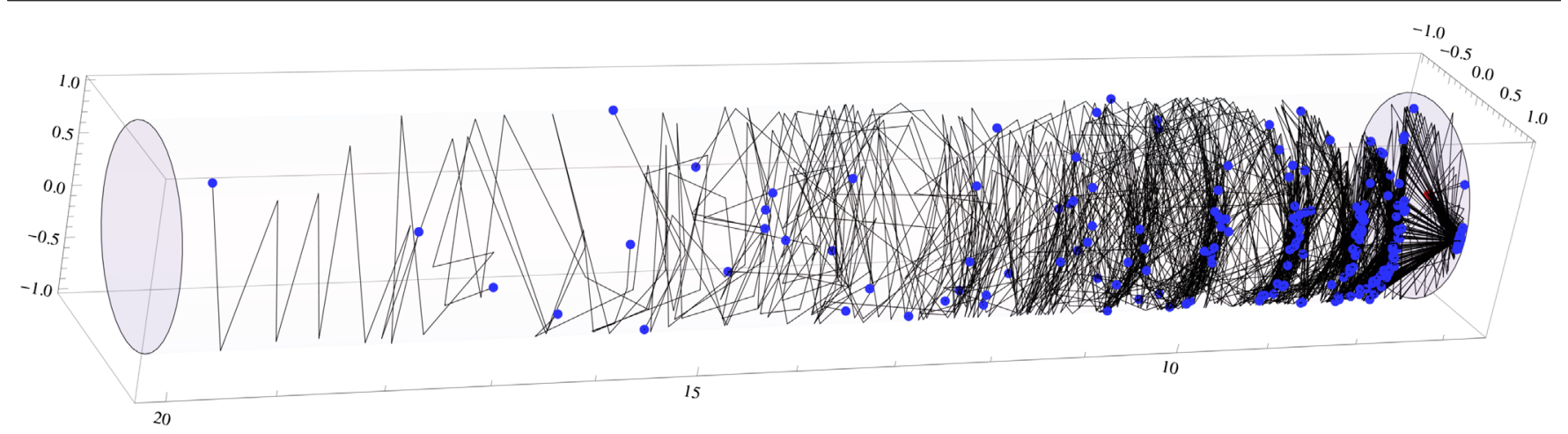

FIG. 12. Photon trajectories from a dipole in three dimensions. The photon source is on the right. For purposes of illustration, the transverse geometry has been distorted from an ellipse to a circle, and the longitudinal dimension has been shrunk by a factor of 10 . Black lines are trajectories, and blue dots are photon absorption sites. Photons generated by the beam propagate downstream (to the left in the figure) and strike the vacuum chamber. Some are absorbed, but most scatter and strike the vacuum chamber further downstream. The absorption site locations tend to be clumped in several clusters (at the location of downstream dipoles), with decreasing intensity as we get further from the source.

description of the derivation of the theory, including the underlying assumptions, which is presented in the Appendix here.

In Sec. III of [20], direct measurements of the surface roughness parameters $\sigma$ and $T$ of two samples of (aluminum) technical accelerator vacuum chamber surfaces were reported. These measurements support the assumptions in the scattering model that the rms surface roughness and the correlation length are the same in both transverse dimensions [see Eq. (A54) and Eq. (A60)]. They also support the use of a Gaussian form for the autocorrelation function.

The measurements, which were done using an atomic force microscope, yielded an average value for the rms surface roughness $\sigma \sim 100 \mathrm{~nm}$. This value was found to be roughly the same in both transverse directions and for both samples, with a variance around $10 \%-25 \%$. The observed autocorrelation function was much closer in form to a Gaussian than an exponential (see Fig. 13 in [20]). The fitted autocorrelation coefficient itself varied widely (from about $3000 \mathrm{~nm}$ to about $17000 \mathrm{~nm}$ ) between the samples and between transverse directions, but the measurement variance was so large $(25 \%-65 \%)$ that the results were statistically consistent with no variation with transverse direction. (see Table I in [20]).

\section{PHOTON PRODUCTION, TRANSPORT, AND ABSORPTION SIMULATION EXAMPLES}

\section{A. Photon emission in a single dipole}

As an example simulation, we consider the CesrTA ring with a $5.3 \mathrm{GeV}$ positron beam. The vacuum chamber is a simple ellipse $9 \mathrm{~cm}$ horizontally by $5 \mathrm{~cm}$ vertically. Figure 12 illustrates the three-dimensional nature of the simulation. In this case, photon generation is restricted to the upstream end of the first dipole on the right in Fig. 12. Photons generated by the beam propagate downstream and strike the vacuum chamber. Some are absorbed here, but most scatter and strike the vacuum chamber further downstream. More are absorbed here, but many others scatter again. Blue dots represent absorption sites. For this simple example, in which the photon source is localized longitudinally, the absorption site locations tend to be clumped in several clusters (at the location of downstream dipoles), with decreasing intensity as we get further from the source.

\section{B. Photon emission throughout the ring, realistic vacuum chamber}

As a second example, photon emission throughout the CesrTA ring from a $2.1 \mathrm{GeV}$ positron beam was simulated with a realistic vacuum chamber profile as shown in Fig. 13. Four different scattering conditions were considered: (a) all specular scattering; (b) specular and diffuse scattering from a polished surface $(\sigma=4 \mathrm{~nm}$, with $T=200 \mathrm{~nm}$ ); specular and diffuse scattering from two different rough surfaces: (c) $\sigma=100 \mathrm{~nm}, T=5500 \mathrm{~nm}$, and (d) $\sigma=200 \mathrm{~nm}, T=5500 \mathrm{~nm}$. Diffuse scattering was simulated, using the model described in Sec. III. Figure 14 shows the absorbed photon distribution (in photons/meter/ radian) vs. polar angle (measured around the vacuum chamber, with zero angle corresponding to the radial outside direction), averaged over four different types of magnetic environment, for the four scattering conditions.

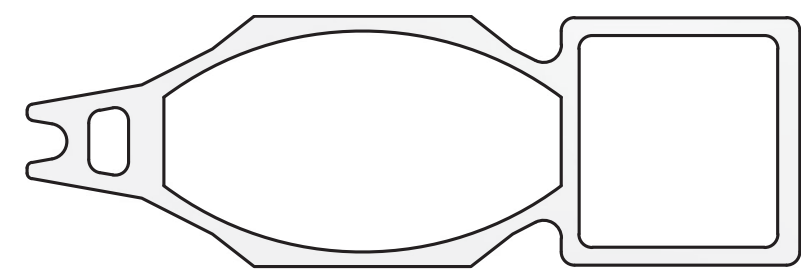

FIG. 13. CesrTA beam pipe cross-section. The vacuum chamber (middle) is $9 \mathrm{~cm}$ wide and $5 \mathrm{~cm}$ high. The cooling water chamber (left) and pump chamber (right) were not part of the simulation. 

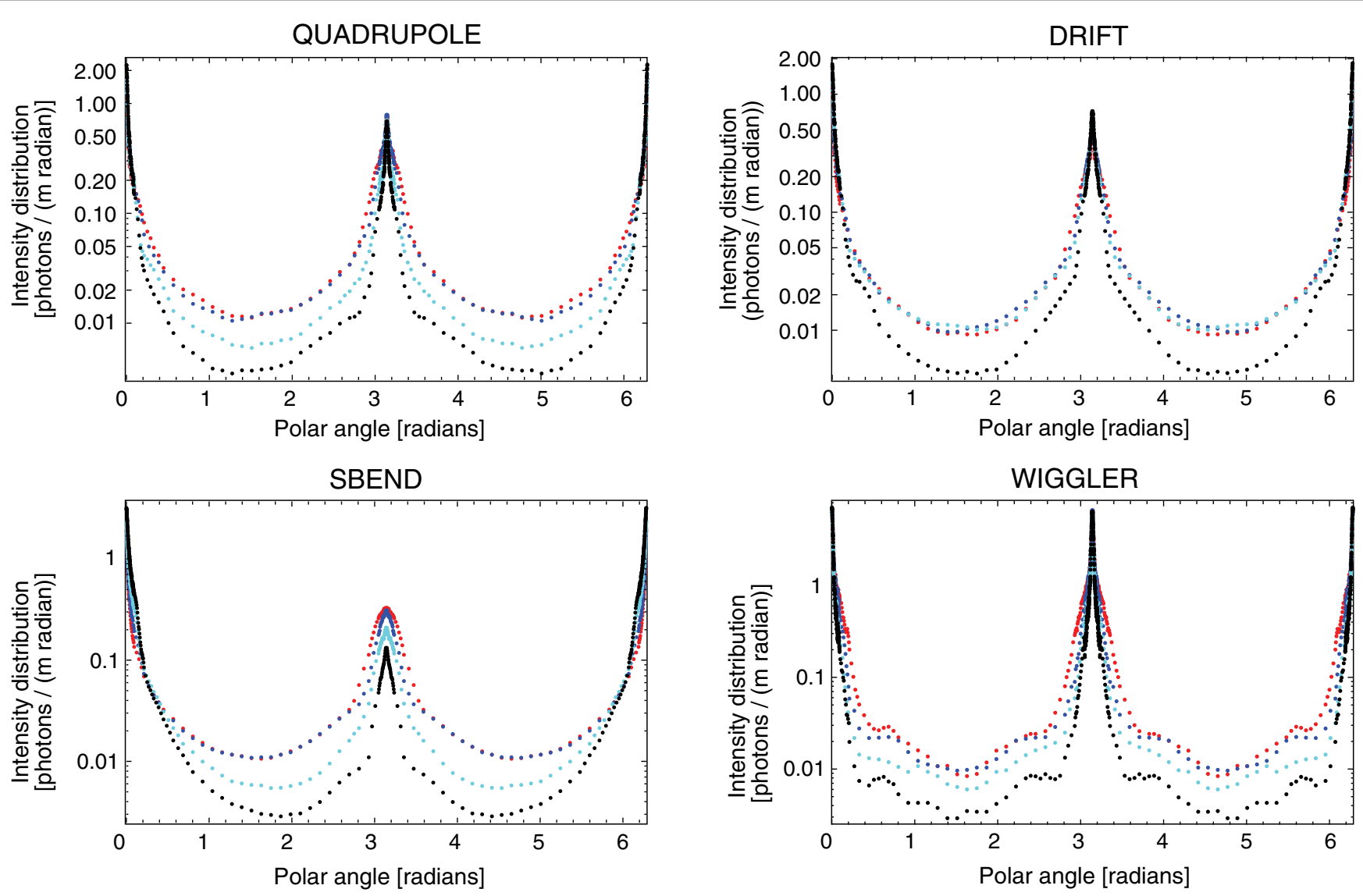

FIG. 14. Absorbed photon distribution vs. polar angle averaged over each type of magnetic environment. Zero angle corresponds to the radial outside direction. The different colors correspond different scattering conditions: (a) Black: pure specular scattering; (b) Cyan: scattering from a polished surface with $\sigma=4 \mathrm{~nm}, T=200 \mathrm{~nm}$; (c) Blue: scattering from a rough surface with $\sigma=100 \mathrm{~nm}$, $T=5500 \mathrm{~nm}$; (d) Red: scattering from a rough surface with $\sigma=200 \mathrm{~nm}, T=5500 \mathrm{~nm}$.

In Fig. 14, comparing the results of all specular reflection [curves (a)] versus the results with specular and diffuse reflection from a rough surface [curves (c) or (d)], it is seen that the photon intensity on the top and bottom of the chamber (polar angles of $\pi / 2$ and $3 \pi / 2$ ) with all specular reflection is much higher than with both specular and diffuse reflection. This is due to the out-of-plane diffuse scattering, which results in substantial amounts of radiation scattering out of the median plane. In addition, the radiation striking the radial inside edge of the vacuum chamber (polar angle near $\pi$ ) is also increased in the SBEND and WIGGLER elements.

Comparing the two rough surfaces cases [curves (c) and (d)], it can be seen that there is not much dependence on $\sigma$. A polished surface [curves (b)] gives considerably less scattering, as expected, but there is still a significant difference between this case and pure specular reflection [curves (a)].

In Fig. 14, the three curves showing absorption with diffusely scattered photons, curves (b), (c), and (d) are very similar. The reason for this is the following. Photons which are absorbed in the drifts have scattered out of the magnetic elements in which they were produced via synchrotron radiation. Since the reflectivity is high for low energy photons with low grazing angles, most of the scattered photons in the drifts will tend to have lower energy and be scattered at low grazing angles, which correlates with wider angular distributions, than in the magnetic elements. The scattered photon angular distribution will be narrower for the smoother surface, case (b), than (c) or (d), but if the distribution is already wide enough in case (b) that many photons hit the top and bottom of the chamber and are absorbed, then a wider distribution for cases (b) and (c) will not make much difference for the absorption rates, and the results for cases (b), (c) and (d) will be similar.

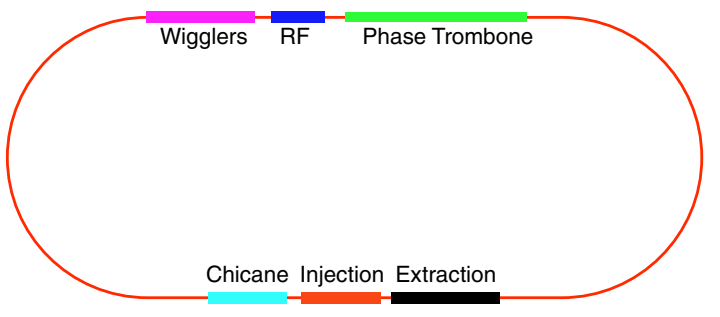

FIG. 15. ILC damping ring schematic layout. 


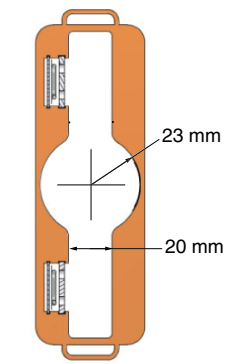

WIGGLER CHAMBER

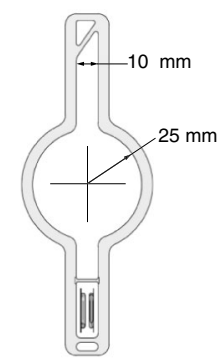

ARC CHAMBER

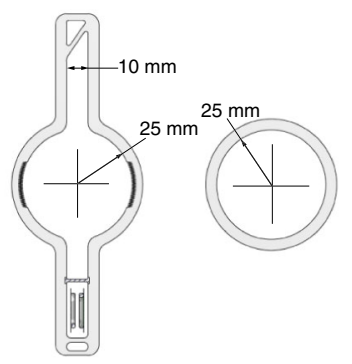

DIPOLE CHAMBER DRIFT CHAMBER
FIG. 16. Vacuum chamber designs for magnetic elements in the ILC damping ring. The upper part of the figures correspond to the outer radius of the ring, when installed.

\section{Application to the ILC damping ring}

As part of the effort [21] to characterize the electron cloud effect in the ILC damping ring, Synrad3D has been used to predict the radiation environment in the vacuum chamber of the ring. The ring layout is shown in Fig. 15. The radiation environment will be different in the arc

regions, where the principal source of photons is the arc dipoles, and the wiggler region, where the wigglers are a source of intense radiation.

Figure 16 shows the design of the vacuum chamber. The radial inside and outside of the wiggler antechamber are designed to fully absorb any photons which strike them. In the arc and dipole antechambers, a slanted wall on the radial outside reduces the scattering of photons back into the chamber.

The absorbed photon distributions predicted by Synrad3D are illustrated in Fig. 17. Top-down symmetry is broken here, because of the backscattering from the slanted wall of the arc and dipole antechambers. The absence of photons at zero and $\pi$ radians are due to the antechambers.

\section{SUMMARY}

As part of the Bmad code ecosystem, a program called Synrad3D has been written to track synchrotron radiation photons generated in storage rings. Synrad3D can handle complicated lattice geometries including nonplanar lattices

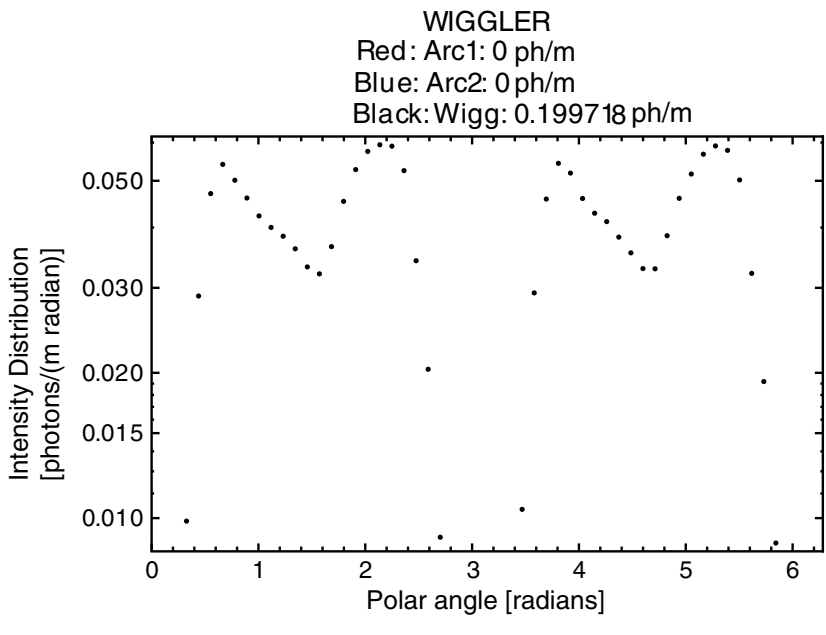

SBEND

Red: Arc1: $0.0158338 \mathrm{ph} / \mathrm{m}$ Blue: Arc2: $0.0160908 \mathrm{ph} / \mathrm{m}$

Red: Arc1: $0.0326384 \mathrm{ph} / \mathrm{m}$

Blue: Arc2: $0.0358304 \mathrm{ph} / \mathrm{m}$

Black: Wigg: $0.220963 \mathrm{ph} / \mathrm{m}$

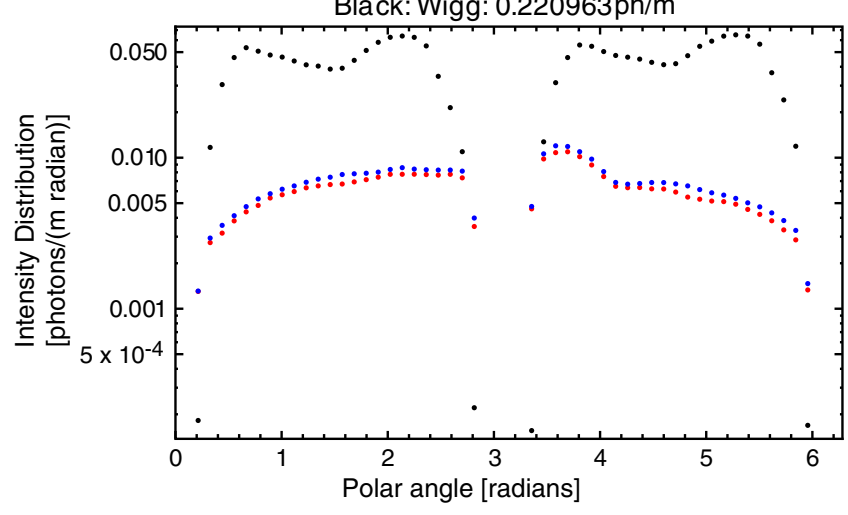

Black: Wigg: $0 \mathrm{ph} / \mathrm{m}$

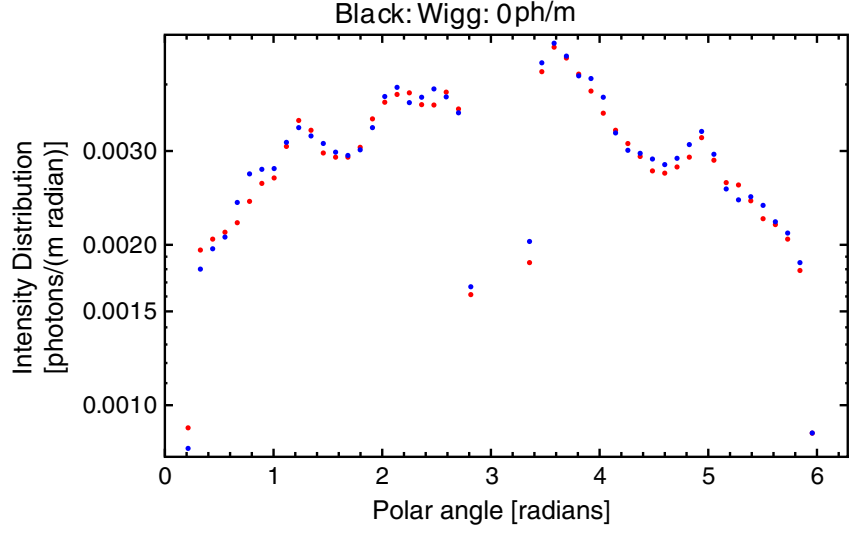

FIG. 17. Photon absorption distributions in different magnetic environments (QUADRUPOLE, WIGGLER, DRIFT and SBEND) and ring regions (Arc1, Arc2, and Wiggler) for the ILC damping ring. 
and intersecting beam lines. Synrad3D can also handle a wide variety of vacuum chamber profiles with varying surface properties.

The program includes scattering from the vacuum chamber walls, based on x-ray data from an LBNL database [15] for the smooth-surface reflectivity, and an analytical model $[13,14]$ for diffuse scattering from a surface with finite roughness.

The predictions of the scattering model have been benchmarked against measurements at DA $\Phi$ NE. Additional benchmarking against recent $\mathrm{x}$-ray scattering measurements has also been reported [20].

Results from the program have given photon absorption site distributions for the CesrTA ring, which have been used as input to electron cloud buildup simulations, whose results can be compared with tune shift [6], retarded field analyzer (RFA), and shielded pickup measurements [22]. The program has also been used to model the radiation environment in the ILC damping ring and the Advanced Photon Source (APS) [23].

\section{ACKNOWLEDGMENTS}

Work supported by grants from the U.S. National Science Foundation (No. PHY-1416318, No. PHY1002467, and No. PHY-0734867), and U.S. Department of Energy (No. DE-FC02-08ER41538).

\section{APPENDIX: DIFFUSE SCATTERING FORMALISM}

This appendix presents the details of the formalism used to describe diffuse scattering. The basic approach is to use scalar Kirchhoff diffraction theory, as originally formulated by Beckmann [13], to describe the angular distribution of power scattered from a random rough surface with a normal distribution variation in height, with $\mathrm{rms} \sigma$, and with a transverse autocorrelation length $T$.

In the material below, we present a derivation of the results from scalar Kirchhoff diffraction theory for a general surface, starting with an expression from Jackson [24].

For the case of a smooth surface, we show how the general expressions for the scattered field and power are in agreement with Jackson [24]. For a random rough surface, we use the general expressions, following the methodology of Beckmann [13], to derive results for the mean value of the scattered power.

We have done this for both a Gaussian and an exponential transverse autocorrelation function. The results are in agreement with Beckmann [13], for the Gaussian autocorrelation function. (Beckmann [13] does not consider the exponential autocorrelation function).

Finally, the scattered power expressions are used to derive marginal and cumulative probability distribution functions for the angular variables. These functions are used in the Synrad3D code to choose outgoing scattering angles from a rough vacuum chamber surface.

\section{General scalar theory}

The notation and terminology are taken from Beckmann [13] (Chap. 3). The starting point is Jackson [24] (p. 491, Eq. 10.108) which gives the Kirchhoff (scalar) field integral. When rewritten in the notation from Beckmann [13] (p. 19, Eq. 8), the integral is

$E_{2}\left(r_{0}\right)=\frac{\mathrm{e}^{\imath k r_{0}}}{4 \pi r_{0}} \int d S \mathrm{e}^{-\imath \mathbf{k}_{2} \cdot \mathbf{r}}\left[-\mathbf{n} \cdot \nabla E_{1}(\mathbf{r})-\imath \mathbf{k}_{\mathbf{2}} \cdot \mathbf{n} E_{1}(\mathbf{r})\right]$

in which $k$ is the photon wave number, $E_{2}\left(r_{0}\right)$ is the (scalar) field at the point with radial coordinate $r_{0}$, resulting from the integral over the whole surface $S$ of the scattered (scalar) field $E_{1}(\mathbf{r}) . \mathbf{r}$ is a point on the surface $S$, the local surface unit normal is $\mathbf{n}$, and the outgoing wave vector $\mathbf{k}_{\mathbf{2}}$ is

$\mathbf{k}_{\mathbf{2}}=k\left(\cos \theta_{2} \hat{\mathbf{z}}+\sin \theta_{2} \cos \phi_{2} \hat{\mathbf{x}}+\sin \theta_{2} \sin \phi_{2} \hat{\mathbf{y}}\right)$.

To evaluate this, we write the scattered field as

$$
E_{1}(\mathbf{r})=\mathcal{R} E_{0} \mathrm{e}^{\mathbf{l} \mathbf{k}_{1} \cdot \mathbf{r}}
$$

Here $E_{0}$ is the incident (scalar) field, and the incident wave vector $\mathbf{k}_{1}$ is

$$
\mathbf{k}_{1}=k\left(-\cos \theta_{1} \hat{\mathbf{z}}+\sin \theta_{1} \hat{\mathbf{x}}\right) .
$$

$\mathcal{R}$ is the smooth-surface field reflection coefficient. The wave vectors and radius vectors used in Eq. (A1) and Eq. (A3) are illustrated in Fig. 18 and Fig. 19.

For the terms in the Kirchhoff integral, we have

$$
\mathbf{n} \cdot \nabla E_{1}(\mathbf{r})=\mathcal{R} E_{0} \mathrm{e}^{\imath \mathbf{k}_{1} \cdot \mathbf{r}} l \mathbf{k}_{1}^{\prime} \cdot \mathbf{n}
$$

in which $\mathbf{k}_{\mathbf{1}}^{\prime}$ is a vector in the direction of the reflected wave from the local surface. Using

$$
\mathbf{k}_{1}^{\prime} \cdot \mathbf{n}=-\mathbf{k}_{\mathbf{1}} \cdot \mathbf{n} .
$$

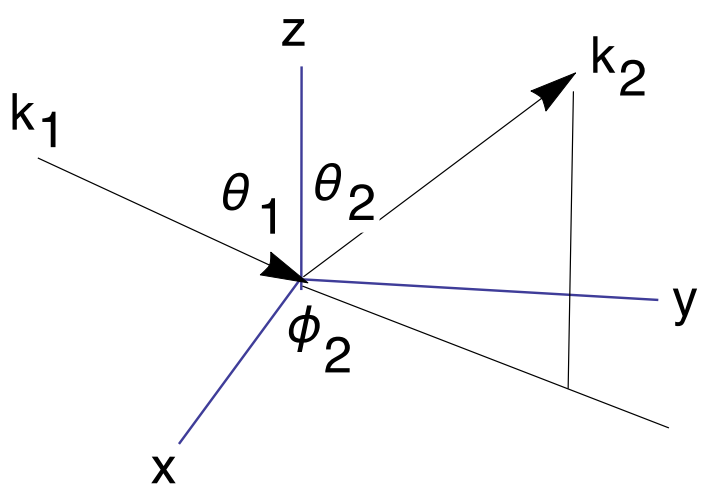

FIG. 18. Coordinate system illustrating the vectors $\mathbf{k}_{\mathbf{1}}$ and $\mathbf{k}_{\mathbf{2}}$. 


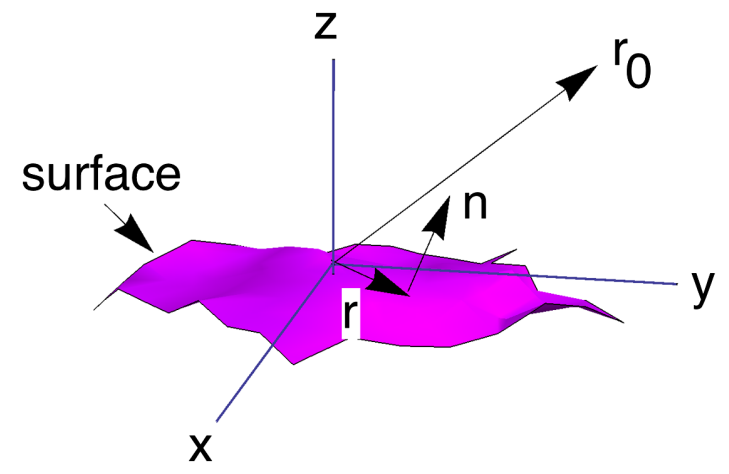

FIG. 19. Coordinate system illustrating the vectors $\mathbf{r}, \mathbf{r}_{\mathbf{0}}$, and $\mathbf{n}$.

then

$$
\mathbf{n} \cdot \nabla E_{1}(\mathbf{r})=-\mathcal{R} E_{0} \mathrm{e}^{\imath \mathbf{k}_{1} \cdot \mathbf{r}} l \mathbf{k}_{\mathbf{1}} \cdot \mathbf{n}
$$

and we have

$-\mathbf{n} \cdot \nabla E_{1}(\mathbf{r})-\imath \mathbf{k}_{\mathbf{2}} \cdot \mathbf{n} E_{1}(\mathbf{r})=\mathcal{R} E_{0} \mathrm{e}^{\imath \mathbf{k}_{1} \cdot \mathbf{r}} \imath\left(\mathbf{k}_{\mathbf{1}}-\mathbf{k}_{\mathbf{2}}\right) \cdot \mathbf{n}$

so

$$
E_{2}\left(r_{0}\right)=\imath E_{0} \frac{\mathrm{e}^{\imath k r_{0}}}{4 \pi r_{0}} \int_{S} \mathrm{e}^{\imath \mathbf{v} \cdot \mathbf{r}} \mathcal{R} \mathbf{v} \cdot \mathbf{n} d S
$$

in which

$$
\begin{aligned}
\mathbf{v}= & \mathbf{k}_{\mathbf{1}}-\mathbf{k}_{\mathbf{2}} \\
= & k\left(\sin \theta_{1}-\sin \theta_{2} \cos \phi_{2}\right) \hat{\mathbf{x}} \\
& -k\left(\cos \theta_{1}+\cos \theta_{2}\right) \hat{\mathbf{z}}-k \sin \theta_{2} \sin \phi_{2} \hat{\mathbf{y}} .
\end{aligned}
$$

To describe the surface from which the scattering occurs, we take the surface height to be a function of the transverse coordinates $x$ and $y$ :

$$
z=\xi(x, y)
$$

The local surface normal vector is

$$
\mathbf{N}=\hat{\mathbf{z}}-\frac{\partial \xi}{\partial x} \hat{\mathbf{x}}-\frac{\partial \xi}{\partial y} \hat{\mathbf{y}}
$$

The local surface normal unit vector is

$$
\mathbf{n}=\frac{\mathbf{N}}{N}=\frac{\hat{\mathbf{z}}-\frac{\partial \xi}{\partial x} \hat{\mathbf{x}}-\frac{\partial \xi}{\partial y} \hat{\mathbf{y}}}{N}
$$

in which

$$
N^{2}=1+\frac{\partial \xi^{2}}{\partial x}+\frac{\partial \xi^{2}}{\partial y}
$$

The radius vector to a point on the surface is

$$
\mathbf{r}=x \hat{\mathbf{x}}+y \hat{\mathbf{y}}+\xi \hat{\mathbf{z}} .
$$

so

$$
\begin{aligned}
N \mathbf{v} \cdot \mathbf{n} & =-v_{x} \frac{\partial \xi}{\partial x}-v_{y} \frac{\partial \xi}{\partial y}+v_{z} \\
\mathbf{v} \cdot \mathbf{r} & =v_{x} x+v_{y} y+v_{z} \xi .
\end{aligned}
$$

The surface area is

$$
\begin{aligned}
d S & =\sqrt{1+\frac{\partial \xi^{2}}{\partial x}+\frac{\partial \xi^{2}}{\partial y}} d x d y \\
& =N d x d y
\end{aligned}
$$

The integral appearing in Eq. (A9) is then

$$
\begin{aligned}
\int_{S} d S \mathrm{e}^{l \mathbf{v} \cdot \mathbf{r}} \mathcal{R} \mathbf{v} \cdot \mathbf{n}= & \int d x d y \mathrm{e}^{l\left(v_{x} x+v_{y} y+v_{z} \xi\right)} \\
& \times \mathcal{R}\left(-v_{x} \frac{\partial \xi}{\partial x}-v_{y} \frac{\partial \xi}{\partial y}+v_{z}\right)
\end{aligned}
$$

So

$$
\begin{aligned}
E_{2}\left(r_{0}\right)= & \iota E_{0} \frac{\mathrm{e}^{l k r_{0}}}{4 \pi r_{0}} \int d x d y \mathrm{e}^{l\left(v_{x} x+v_{y} y+v_{z} \xi\right)} \\
& \times \mathcal{R}\left(-v_{x} \frac{\partial \xi}{\partial x}-v_{y} \frac{\partial \xi}{\partial y}+v_{z}\right) .
\end{aligned}
$$

It is convenient to transform the integration variables to cylindrical coordinates at this point, defined by

$$
\begin{gathered}
\rho^{2}=x^{2}+y^{2} \\
\tan \phi^{\prime}=\frac{y}{x} .
\end{gathered}
$$

Then

$$
\begin{gathered}
\frac{\partial \xi}{\partial x}=\cos \phi^{\prime} \frac{\partial \xi}{\partial \rho}-\frac{\sin \phi^{\prime}}{\rho} \frac{\partial \xi}{\partial \phi^{\prime}} \\
\frac{\partial \xi}{\partial y}=\sin \phi^{\prime} \frac{\partial \xi}{\partial \rho}+\frac{\cos \phi^{\prime}}{\rho} \frac{\partial \xi}{\partial \phi^{\prime}}
\end{gathered}
$$

Introducing

$$
\begin{gathered}
v_{t}^{2}=v_{x}^{2}+v_{y}^{2} \\
\tan \phi_{v}=\frac{v_{y}}{v_{x}}
\end{gathered}
$$


we have

$$
\begin{aligned}
& v_{x} \frac{\partial \xi}{\partial x}+v_{y} \frac{\partial \xi}{\partial y} \\
& =v_{t}\left[\frac{\partial \xi}{\partial \rho} \cos \left(\phi^{\prime}-\phi_{v}\right)-\frac{\sin \left(\phi^{\prime}-\phi_{v}\right)}{\rho} \frac{\partial \xi}{\partial \phi^{\prime}}\right]
\end{aligned}
$$

and

$$
\mathrm{e}^{l\left(v_{x} x+v_{y} y+v_{z} \xi\right)}=\mathrm{e}^{l\left[v_{t} \rho \cos \left(\phi^{\prime}-\phi_{v}\right)+v_{z} \xi\right]}
$$

so

$$
\begin{aligned}
E_{2}\left(r_{0}\right)= & \iota E_{0} \frac{\mathrm{e}^{\imath k r_{0}}}{4 \pi r_{0}} \int \rho d \rho d \phi^{\prime} \mathrm{e}^{\imath\left[v_{t} \rho \cos \left(\phi^{\prime}-\phi_{v}\right)+v_{z} \xi\right]} \\
& \times \mathcal{R}\left[-\frac{\partial \xi}{\partial \rho} v_{t} \cos \left(\phi^{\prime}-\phi_{v}\right)\right. \\
& \left.+\frac{v_{t} \sin \left(\phi^{\prime}-\phi_{v}\right)}{\rho} \frac{\partial \xi}{\partial \phi^{\prime}}+v_{z}\right]
\end{aligned}
$$

We can simplify this expression with the substitution

$$
\phi=\phi^{\prime}-\phi_{v}
$$

if we assume axial symmetry for $\xi$ :

$$
\xi\left(\rho, \phi^{\prime}\right)=\xi\left(\rho, \phi^{\prime}-\phi_{v}\right)
$$

Then

$$
\begin{aligned}
E_{2}\left(r_{0}\right)= & \iota E_{0} \frac{\mathrm{e}^{\imath k r_{0}}}{4 \pi r_{0}} \int \rho d \rho d \phi \mathrm{e}^{l\left(v_{t} \rho \cos \phi+v_{z} \xi\right)} \\
& \times \mathcal{R}\left(-\frac{\partial \xi}{\partial \rho} v_{t} \cos \phi+\frac{v_{t} \sin \phi}{\rho} \frac{\partial \xi}{\partial \phi}+v_{z}\right)
\end{aligned}
$$

\section{Scattering from a smooth surface}

\section{a. Field}

If the surface is perfectly smooth, then

$$
\xi=\frac{\partial \xi}{\partial \rho}=\frac{\partial \xi}{\partial \phi}=0
$$

and we have

$$
E_{2}\left(r_{0}\right)=\imath E_{0} \frac{\mathrm{e}^{\imath k r_{0}}}{4 \pi r_{0}} v_{z} \mathcal{R} \int \rho d \rho d \phi \mathrm{e}^{\imath v_{t} \rho \cos \phi}
$$

The field reflectivity $\mathcal{R}$ has been removed from the integral, as it is independent of position for a flat surface. If we take the scattering surface to be a disk of radius $a$, and surface area $A=\pi a^{2}$, then

$$
\begin{aligned}
\int \rho d \rho d \phi \mathrm{e}^{l v_{t} \rho \cos \phi} & =\int_{0}^{a} \rho d \rho \int_{0}^{2 \pi} d \phi \mathrm{e}^{i v_{t} \rho \cos \phi} \\
& =2 \pi \int_{0}^{a} \rho d \rho J_{0}\left(\rho v_{t}\right) \\
& =\frac{2 \pi a J_{1}\left(a v_{t}\right)}{v_{t}}
\end{aligned}
$$

in which $J_{0}(x)$ and $J_{1}(x)$ are Bessel functions. Thus

$$
\begin{aligned}
E_{2}\left(r_{0}\right) & =\imath E_{0} \frac{\mathrm{e}^{\imath k r_{0}}}{4 \pi r_{0}} v_{z} \mathcal{R} \frac{2 \pi a J_{1}\left(a v_{t}\right)}{v_{t}} \\
& =\imath A v_{z} \mathcal{R} E_{0} \frac{\mathrm{e}^{\imath k r_{0}}}{2 \pi r_{0}} \frac{J_{1}\left(a v_{t}\right)}{a v_{t}}
\end{aligned}
$$

in which

$$
v_{z}=-k\left(\cos \theta_{1}+\cos \theta_{2}\right)
$$

and

$$
\begin{aligned}
v_{t}^{2} & =v_{x}^{2}+v_{y}^{2} \\
& =k^{2}\left[\left(\sin \theta_{1}-\sin \theta_{2} \cos \phi_{2}\right)^{2}+\left(\sin \theta_{2} \sin \phi_{2}\right)^{2}\right] \\
& =k^{2}\left(\sin \theta_{1}^{2}+\sin \theta_{2}^{2}-2 \cos \phi_{2} \sin \theta_{1} \sin \theta_{2}\right) .
\end{aligned}
$$

\section{b. Power}

According to Jackson [24], the consistent way to use the scalar electric field to compute the power is to use the relation

$$
\frac{d P_{2}}{d \Omega_{2}}=\frac{r_{0}^{2}}{2 Z_{0}}\left|E_{2}\left(r_{0}\right)\right|^{2} .
$$

Thus

$$
\frac{d P_{2}}{d \Omega_{2}}=\frac{A^{2} v_{z}^{2} \mathcal{R}^{2}\left|E_{0}\right|^{2}}{\left(2 Z_{0}\right) 4 \pi^{2}}\left[\frac{J_{1}\left(a v_{t}\right)}{a v_{t}}\right]^{2} .
$$

The total power in the incident field striking the area $A$ is

$$
P_{0}=\frac{A}{2 Z_{0}}\left|\hat{\mathbf{z}} \cdot \hat{\mathbf{k}}_{\mathbf{1}}\right|\left|\mathbf{E}_{0}\right|^{2}=\frac{A \cos \theta_{1}}{2 Z_{0}}\left|E_{0}\right|^{2}
$$

so

$$
\left.\frac{1}{P_{0}} \frac{d P_{2}}{d \Omega_{2}}\right|_{\text {spec, flat }}=\frac{A v_{z}^{2} \mathcal{R}^{2}}{4 \pi^{2} \cos \theta_{1}}\left[\frac{J_{1}\left(a v_{t}\right)}{a v_{t}}\right]^{2} .
$$

This represents the specular power reflected from a flat disk of radius $a$ and field reflectivity $\mathcal{R}$. The Bessel function dependence represents diffraction from the edge of the disk. If we take the limit $a \rightarrow \infty$, the diffraction effects vanish, and the Bessel function dependence makes the power sharply 
peaked at the point $v_{t}=0$, corresponding to the specular condition on the scattering angles: $\sin \theta_{2}=\sin \theta_{1}$ and $\phi_{2}=0$. In this case, integration of this expression over the scattering angles yields the total scattering probability $P_{\text {scatt }}$ :

$$
P_{\text {scatt }}=\left.\lim _{a \rightarrow \infty} \int d \Omega_{2} \frac{1}{P_{0}} \frac{d P_{2}}{d \Omega_{2}}\right|_{\text {spec, flat }}=\mathcal{R}^{2}=R
$$

in which we introduce the smooth-surface photon (or intensity) reflectivity $R$, which is related to the field reflectivity $\mathcal{R}$ by

$$
R=\mathcal{R}^{2}
$$

\section{Scattering from a rough surface \\ a. Field}

For a rough surface, we use the following relations in Eq. (A31):

$$
\begin{aligned}
\int \rho & d \rho \mathcal{R} \mathrm{e}^{l\left(v_{t} \rho \cos \phi+v_{z} \xi\right)} \frac{\partial \xi}{\partial \rho} \\
= & \left.\frac{\mathcal{R} \rho}{l v_{z}} \mathrm{e}^{l\left(v_{t} \rho \cos \phi+v_{z} \xi\right)}\right|_{\rho=0} ^{\rho=a} \\
& -\frac{1}{l v_{z}} \int d \rho \mathcal{R} \mathrm{e}^{l\left(v_{t} \rho \cos \phi+v_{z} \xi\right)}\left(1+\imath v_{t} \rho \cos \phi\right) \\
\int d \phi & \mathcal{R} \mathrm{e}^{l\left(v_{t} \rho \cos \phi+v_{z} \xi\right)} \frac{\partial \xi}{\partial \phi} \sin \phi \\
= & \left.\frac{\mathcal{R} \sin \phi}{l v_{z}} \mathrm{e}^{l\left(v_{t} \rho \cos \phi+v_{z} \xi\right)}\right|_{\phi=0} ^{\phi=2 \pi} \\
& -\frac{1}{l v_{z}} \int d \rho \mathcal{R} \mathrm{e}^{l\left(v_{t} \rho \cos \phi+v_{z} \xi\right)}\left(\cos \phi-l v_{t} \rho \sin ^{2} \phi\right) .
\end{aligned}
$$

$$
\begin{aligned}
& \text { So } \\
& \begin{aligned}
E_{2}\left(r_{0}\right)= & -E_{0} \frac{\mathrm{e}^{l k r_{0}}}{4 \pi r_{0}} \frac{1}{v_{z}}\left(\int d \rho d \phi \mathcal{R} \mathrm{e}^{l\left(v_{t} \rho \cos \phi+v_{z} \xi\right)}\right. \\
& \times\left[-\left(1+\imath v_{t} \rho \cos \phi\right) v_{t} \cos \phi+v_{t}\left(\cos \phi-\imath v_{t} \rho \sin ^{2} \phi\right)\right. \\
& \left.\left.-\imath \rho v_{z}^{2}\right]+a v_{t} \int_{0}^{2 \pi} d \phi \cos \phi \mathcal{R} \mathrm{e}^{l\left[v_{t} a \cos \phi+v_{z} \xi(a, \phi)\right]}\right) \\
= & \iota E_{0} \frac{\mathrm{e}^{\imath k r_{0}}}{4 \pi r_{0}} \frac{v_{t}^{2}+v_{z}^{2}}{v_{z}}\left(\int \rho d \rho d \phi \mathcal{R} \mathrm{e}^{l\left(v_{t} \rho \cos \phi+v_{z} \xi\right)}\right. \\
& \left.+\frac{l a v_{t}}{v_{t}^{2}+v_{z}^{2}} \int_{0}^{2 \pi} d \phi \cos \phi \mathcal{R} \mathrm{e}^{l\left[v_{t} a \cos \phi+v_{z} \xi(a, \phi)\right]}\right) .
\end{aligned}
\end{aligned}
$$

The last term in the final bracket in Eq. (A46) is evaluated on the boundary of the scattering region. This term is typically neglected in the computation of radiant power scattered from rough surfaces (see Beckmann [13]). As noted in Ogilvy [14], p. 82, neglecting the boundary term is correct only for the diffusely scattered power, not for the specular power. In this application, we are only interested in the expression for the diffusely scattered power, so we will neglect this term.

Then, using

$$
v^{2}=v_{t}^{2}+v_{z}^{2}
$$

the field equation is

$E_{2}\left(r_{0}\right)=\iota_{0} E_{0} \frac{\mathrm{e}^{\imath k r_{0}}}{4 \pi r_{0}} \frac{v^{2}}{v_{z}} \int \rho d \rho d \phi \mathcal{R} \mathrm{e}^{l\left(v_{t} \rho \cos \phi+v_{z} \xi\right)}$.

\section{b. Power}

The power is

$$
\begin{aligned}
\frac{d P_{2}}{d \Omega_{2}} & =\frac{r_{0}^{2}}{2 Z_{0}}\left|E_{2}\left(r_{0}\right)\right|^{2} \\
& =\frac{\left|E_{0}\right|^{2}}{\left(2 Z_{0}\right)(4 \pi)^{2}} \frac{v^{4}}{v_{z}^{2}} \int \rho d \rho d \phi \rho^{\prime} d \rho^{\prime} d \phi^{\prime} \mathcal{R} \mathcal{R}^{\prime} \mathrm{e}^{i \eta\left(\rho, \rho^{\prime}, \phi, \phi^{\prime}\right)} \\
& =\frac{P_{0}}{(4 \pi)^{2} A \cos \theta_{1}} \frac{v^{4}}{v_{z}^{2}} \int \rho d \rho d \phi \rho^{\prime} d \rho^{\prime} d \phi^{\prime} \mathcal{R} \mathcal{R}^{\prime} \mathrm{e}^{i \eta\left(\rho, \rho^{\prime}, \phi, \phi^{\prime}\right)}
\end{aligned}
$$

in which

$\eta\left(\rho, \rho^{\prime}, \phi, \phi^{\prime}\right)=v_{t}\left(\rho \cos \phi-\rho^{\prime} \cos \phi^{\prime}\right)+v_{z}\left(\xi-\xi^{\prime}\right)$

with $\xi(\rho, \phi)=\xi$ and $\xi\left(\rho^{\prime}, \phi^{\prime}\right)=\xi^{\prime}$.

The field reflection coefficient $\mathcal{R}$ depends on the incident photon energy $k$, the incident polar angle $\theta_{1}$, and the local surface slopes $\frac{\partial \xi}{\partial x}$ and $\frac{\partial \xi}{\partial y}$. The dependence of $\mathcal{R}$ on the local slopes means that, in general, $\mathcal{R}$ cannot be taken out of the integral. However, since the local surface slopes are typically small, the conventional treatment (as in Beckmann [13]) is to ignore this dependence, and treat $\mathcal{R}$ as a function of $k$ and $\theta_{1}$ only. We can then take it outside the integral and introduce the photon reflectivity from Eq. (A43):

$$
\mathcal{R R}^{\prime} \rightarrow \mathcal{R}^{2}=R
$$

With this replacement, the power is

$\frac{d P_{2}}{d \Omega_{2}}=\frac{P_{0} R}{(4 \pi)^{2} A \cos \theta_{1}} \frac{v^{4}}{v_{z}^{2}} \int \rho d \rho d \phi \rho^{\prime} d \rho^{\prime} d \phi^{\prime} \mathrm{e}^{i \eta\left(\rho, \rho^{\prime}, \phi, \phi^{\prime}\right)}$. 
To proceed with the evaluation of the power, we must specify the surface height function $\xi(\rho, \phi)$. The conventional approach is to take the surface height $\xi$ to be a random variable. This variable can be described by specifying its statistical measures: the distribution function and the characteristic function.

Let the two-dimensional distribution function of the random variable $\xi$ be $w\left(\xi_{1}, \xi_{2}\right)$. The two-dimensional characteristic function of $\xi$ is

$$
\begin{aligned}
\chi(u, v) & =\int d \xi_{1} d \xi_{2} w\left(\xi_{1}, \xi_{2}\right) \mathrm{e}^{l\left(u \xi_{1}+v \xi_{2}\right)} \\
& =\left\langle\mathrm{e}^{l\left(u \xi_{1}+v \xi_{1}\right)}\right\rangle
\end{aligned}
$$

in which the brackets specify an ensemble average over the distribution function.

For this analysis, we assume a Gaussian distribution of surface fluctuations. The normalized two-dimensional distribution function is

$$
w\left(\xi_{1}, \xi_{2}\right)=\frac{1}{2 \pi \sigma^{2} \sqrt{1-C^{2}}} \mathrm{e}^{-\frac{\xi_{1}^{2}-2 C \xi_{1} \xi_{2}+\xi_{2}^{2}}{2 \sigma^{2}\left(1-C^{2}\right)}}
$$

in which

$$
\sigma^{2}=\left\langle\xi_{1}^{2}\right\rangle=\left\langle\xi_{2}^{2}\right\rangle
$$

is the mean square surface height fluctuation (assumed to be the same in both transverse directions), and

$$
C=\frac{\left\langle\xi_{1} \xi_{2}\right\rangle}{\sigma^{2}}
$$

is the autocorrelation (which is typically a function of the transverse coordinates). From Eq. (A52), the characteristic function is

$$
\chi(u, v)=\mathrm{e}^{-\frac{\sigma^{2}}{2}\left(u^{2}+v^{2}+2 C u v\right)} .
$$

We can write the ensemble average of the power in terms of the characteristic function for $\xi$ as follows:

$$
\begin{aligned}
\left\langle\frac{d P_{2}}{d \Omega_{2}}\right\rangle= & P_{0} \frac{R}{(4 \pi)^{2} A \cos \theta_{1}} \frac{v^{4}}{v_{z}^{2}} \\
& \times \int \rho d \rho d \phi \rho^{\prime} d \rho^{\prime} d \phi^{\prime}\left\langle\mathrm{e}^{i \eta\left(\rho, \rho^{\prime}, \phi, \phi^{\prime}\right)}\right\rangle
\end{aligned}
$$

in which

$$
\begin{aligned}
\left\langle\mathrm{e}^{i \eta\left(\rho, \rho^{\prime}, \phi, \phi^{\prime}\right)}\right\rangle & =\mathrm{e}^{i v_{t}\left(\rho \cos \phi-\rho^{\prime} \cos \phi^{\prime}\right)}\left\langle\mathrm{e}^{l v_{z}\left(\xi-\xi^{\prime}\right)}\right\rangle \\
& =\mathrm{e}^{l v_{t}\left(\rho \cos \phi-\rho^{\prime} \cos \phi^{\prime}\right)} \chi\left(v_{z},-v_{z}\right) \\
& =\mathrm{e}^{i v_{t}\left(\rho \cos \phi-\rho^{\prime} \cos \phi^{\prime}\right)} \mathrm{e}^{-\sigma^{2} v_{z}^{2}(1-C)} .
\end{aligned}
$$

Thus

$$
\begin{aligned}
& \int \rho d \rho d \phi \rho^{\prime} d \rho^{\prime} d \phi^{\prime}\left\langle\mathrm{e}^{i \eta\left(\rho, \rho^{\prime}, \phi, \phi^{\prime}\right)}\right\rangle \\
& =\int \rho d \rho d \phi \rho^{\prime} d \rho^{\prime} d \phi^{\prime} \mathrm{e}^{-g(1-C)} \mathrm{e}^{l v_{t}\left(\rho \cos \phi-\rho^{\prime} \cos \phi^{\prime}\right)}
\end{aligned}
$$

in which

$$
g=\sigma^{2} v_{z}^{2}
$$

Since $C$ is a function of the transverse coordinates, we cannot remove the term containing $C$ from the integral. We assume that the dependence of $C$ on the transverse coordinates has the form in which

$$
C=C\left(\left|\vec{\rho}-\vec{\rho}^{\prime}\right| / T\right)
$$

in which $T$ is the correlation length. By virtue of the substitution in Eq. (A30), the vector $\vec{v}_{t}$ lies along the $x$-axis, so

$$
v_{t}\left(\rho \cos \phi-\rho^{\prime} \cos \phi^{\prime}\right)=\vec{v}_{t} \cdot\left(\vec{\rho}-\overrightarrow{\rho^{\prime}}\right)
$$

so

$$
\begin{aligned}
& \int \rho d \rho d \phi \rho^{\prime} d \rho^{\prime} d \phi^{\prime}\left\langle\mathrm{e}^{i \eta\left(\rho, \rho^{\prime}, \phi, \phi^{\prime}\right)}\right\rangle \\
& =\int d \vec{\rho} d \overrightarrow{\rho^{\prime}} \mathrm{e}^{-g\left(1-C\left(\left|\vec{\rho}-\vec{\rho}^{\prime}\right| / T\right)\right)} \mathrm{e}^{l \vec{l} \vec{v}_{t} \cdot\left(\vec{\rho}-\vec{\rho}^{\prime}\right)} .
\end{aligned}
$$

Let us introduce the new dummy integration variable $\vec{r}$ [not to be confused with the vector defined in Eq. (A15)]

$$
\vec{r}=\overrightarrow{\rho^{\prime}}-\vec{\rho}
$$

then

$$
\begin{gathered}
\int \rho d \rho d \phi \rho^{\prime} d \rho^{\prime} d \phi^{\prime}\left\langle\mathrm{e}^{l \eta\left(\rho, \rho^{\prime}, \phi, \phi^{\prime}\right)}\right\rangle \\
=\int d \vec{\rho} d \overrightarrow{\rho^{\prime}} \mathrm{e}^{-g[1-C(r / T)]} \mathrm{e}^{-l \overrightarrow{v_{t}} \cdot \vec{r}} .
\end{gathered}
$$

We make a power series expansion of the integrand using:

$$
\mathrm{e}^{g C(r / T)}=1+\sum_{m=1}^{\infty} \frac{[g C(r / T)]^{m}}{m !} .
$$

Substituting this, we have 


$$
\begin{aligned}
\int \rho d \rho d \phi \rho^{\prime} d \rho^{\prime} d \phi^{\prime}\left\langle\mathrm{e}^{l \eta\left(\rho, \rho^{\prime}, \phi, \phi^{\prime}\right)}\right\rangle \\
=\mathrm{e}^{-g} \times\left\{\int d \vec{\rho} d \overrightarrow{\rho^{\prime}} \mathrm{e}^{-l \overrightarrow{t_{t}} \cdot \vec{r}}\right. \\
\left.\quad+\sum_{m=1}^{\infty} \int d \vec{\rho} d \overrightarrow{\rho^{\prime}} \frac{[g C(r / T)]^{m}}{m !} \mathrm{e}^{-l \vec{v}_{t} \cdot \vec{r}}\right\} .
\end{aligned}
$$

Using the result from Eq. (A34), we can write the first term in the bracket in Eq. (A66) as

$$
\int d \vec{\rho} d \overrightarrow{\rho^{\prime}} \overrightarrow{\mathrm{v}}_{t} \cdot\left(\vec{\rho}-\vec{\rho}^{\prime}\right)=\left[\frac{2 \pi a J_{1}\left(a v_{t}\right)}{v_{t}}\right]^{2} .
$$

To evaluate the terms in the sum, we note that the correlation function $C$ as a function of $r$ typically falls from 1 , at $r=0$, to zero on the distance scale of the correlation length $T$. Ultimately we will take the radius $a$ of the scattering region to go to infinity, so $T \ll a$. Since the integrand is essentially zero at values of $|r| \gg T \ll a$, we can extend the range of integration over $\vec{r}$ to infinity. Introducing the azimuthal angle $\beta$ associated with $\vec{r}$ in cylindrical coordinates, and noting that, for fixed $\vec{\rho}$,

$$
d \overrightarrow{\rho^{\prime}}=d \vec{r}=r d r d \beta
$$

we have

$$
\begin{aligned}
& \int d \vec{\rho} d \overrightarrow{\rho^{\prime}} \frac{[g C(r / T)]^{m}}{m !} \mathrm{e}^{-l \vec{v}_{t} \cdot \vec{r}} \\
& \approx \int d \vec{\rho} \int_{0}^{\infty} r d r \frac{[g C(r / T)]^{m}}{m !} \int_{0}^{2 \pi} d \beta \mathrm{e}^{-l v_{t} r \cos \beta}
\end{aligned}
$$

Then, using

$$
\begin{gathered}
\int d \vec{\rho}=A \\
\int_{0}^{2 \pi} d \beta \mathrm{e}^{-l v_{t} r \cos \beta}=2 \pi J_{0}\left(r v_{t}\right),
\end{gathered}
$$

in which $A=\pi a^{2}$ is the area of the scattering region, we have

$$
\begin{aligned}
& \int d \vec{\rho} d \overrightarrow{\rho^{\prime}} \frac{[g C(r / T)]^{m}}{m !} \mathrm{e}^{-l \vec{v}_{t} \cdot \vec{r}} \\
& \quad \approx 2 \pi A \int_{0}^{\infty} r d r \frac{[g C(r / T)]^{m}}{m !} J_{0}\left(r v_{t}\right) .
\end{aligned}
$$

Thus

$$
\begin{aligned}
\int \rho d \rho d \phi \rho^{\prime} d \rho^{\prime} d \phi^{\prime}\left\langle\mathrm{e}^{m\left(\rho, \rho^{\prime}, \phi, \phi^{\prime}\right)}\right\rangle \\
\approx 2 \pi A \mathrm{e}^{-g}\left\{2 a^{2}\left[\frac{J_{1}\left(a v_{t}\right)}{a v_{t}}\right]^{2}\right. \\
\left.\quad+\sum_{m=1}^{\infty} \int_{0}^{\infty} r d r \frac{[g C(r / T)]^{m}}{m !} J_{0}\left(r v_{t}\right)\right\} .
\end{aligned}
$$

The power is then

$$
\begin{aligned}
\left\langle\frac{d P_{2}}{d \Omega_{2}}\right\rangle \approx & P_{0} \frac{R e^{-g}}{4 \pi \cos \theta_{1}} \frac{v^{4}}{v_{z}^{2}} \times\left\{\frac{A}{\pi}\left[\frac{J_{1}\left(a v_{t}\right)}{a v_{t}}\right]^{2}\right. \\
& \left.+\frac{1}{2} \sum_{m=1}^{\infty} \int_{0}^{\infty} r d r \frac{[g C(r / T)]^{m}}{m !} J_{0}\left(r v_{t}\right)\right\} .
\end{aligned}
$$

If the surface is smooth, $\sigma=0 \Rightarrow g=0$, the sum disappears in the expression for the power, and we have

$$
\left.\left\langle\frac{d P_{2}}{d \Omega_{2}}\right\rangle\right|_{\sigma=0} \approx P_{0} \frac{A R}{4 \pi^{2} \cos \theta_{1}} \frac{v^{4}}{v_{z}^{2}}\left[\frac{J_{1}\left(a v_{t}\right)}{a v_{t}}\right]^{2} .
$$

In the limit $a \rightarrow \infty$, we have $v_{t} \rightarrow 0$ so $v=v_{z}$ this expression reduces to the specular power from a flat surface, Eq. (A41). Thus, in the limit $a \rightarrow \infty$, we can write

$$
\left.\left\langle\frac{d P_{2}}{d \Omega_{2}}\right\rangle \approx e^{-g} \frac{d P_{2}}{d \Omega_{2}}\right|_{\text {spec,flat }}+\left.\frac{d P_{2}}{d \Omega_{2}}\right|_{\text {diff }}
$$

in which

$$
\begin{aligned}
\left.\frac{d P_{2}}{d \Omega_{2}}\right|_{\text {diff }}= & P_{0} \frac{R e^{-g}}{8 \pi \cos \theta_{1}} \frac{v^{4}}{v_{z}^{2}} \\
& \times \sum_{m=1}^{\infty} \int_{0}^{\infty} r d r \frac{[g C(r / T)]^{m}}{m !} J_{0}\left(r v_{t}\right)
\end{aligned}
$$

Integration over the scattering angles, the total scattering probability from a rough surface can be written as

$$
\begin{aligned}
P_{\text {scatt }} & =R=\lim _{a \rightarrow \infty} \int \frac{d \Omega_{2}}{P_{0}}\left\langle\frac{d P_{2}}{d \Omega_{2}}\right\rangle \\
& \left.\approx e^{-g} \int \frac{d \Omega_{2}}{P_{0}} \frac{d P_{2}}{d \Omega_{2}}\right|_{\text {spec,flat }}+\left.\int \frac{d \Omega_{2}}{P_{0}} \frac{d P_{2}}{d \Omega_{2}}\right|_{\text {diff }} \\
& =P_{\text {spec }}+P_{\text {diff }}
\end{aligned}
$$

in which

$$
P_{\mathrm{spec}}=\left.e^{-g} \int \frac{d \Omega_{2}}{P_{0}} \frac{d P_{2}}{d \Omega_{2}}\right|_{\text {spec,flat }}=e^{-g} R
$$

is the probability of specular reflection, and 
$P_{\mathrm{diff}}=\left.\int \frac{d \Omega_{2}}{P_{0}} \frac{d P_{2}}{d \Omega_{2}}\right|_{\text {diff }}=P_{\mathrm{scatt}}-P_{\mathrm{spec}}=\left(1-e^{-g}\right) R$

is the probability of diffuse reflection.

\section{c. Diffuse power evaluation: Gaussian autocorrelation function}

For a Gaussian autocorrelation function, we have

$$
C(r / T)=\mathrm{e}^{-r^{2} / T^{2}},
$$

so the diffuse power is

$$
\begin{aligned}
\left.\frac{d P_{2}}{d \Omega_{2}}\right|_{\text {diff }}= & P_{0} \frac{R e^{-g}}{8 \pi \cos \theta_{1}} \frac{v^{4}}{v_{z}^{2}} \\
& \times \sum_{m=1}^{\infty} \int_{0}^{\infty} r d r \frac{g^{m} \mathrm{e}^{-m r^{2} / T^{2}}}{m !} J_{0}\left(r v_{t}\right) .
\end{aligned}
$$

The integral is

$$
\int_{0}^{\infty} r d r \mathrm{e}^{-m r^{2} / T^{2}} J_{0}\left(r v_{t}\right)=\frac{T^{2}}{2 m} \mathrm{e}^{-T^{2} v_{t}^{2} / 4 m}
$$

so the power is

$$
\left.\frac{d P_{2}}{d \Omega_{2}}\right|_{\text {diff }}=P_{0} \frac{R e^{-g}}{8 \pi \cos \theta_{1}} \frac{v^{4}}{v_{z}^{2}} \frac{T^{2}}{2} \sum_{m=1}^{\infty} X_{m}
$$

in which

$$
X_{m}=\frac{g^{m}}{m ! m} \mathrm{e}^{-\frac{v_{t}^{2} T^{2}}{4 m}} .
$$

Introducing

$$
\begin{gathered}
x=\cos \theta_{2} \\
y=\cos \theta_{1} \\
\zeta=\cos \phi_{2} \\
h=\sin \theta_{1} \sin \theta_{2}=\sqrt{1-x^{2}} \sqrt{1-y^{2}},
\end{gathered}
$$

we have

$$
\begin{gathered}
v^{4}=4 k^{4}\left(1+\cos \theta_{1} \cos \theta_{2}-\sin \theta_{1} \sin \theta_{2} \cos \phi_{2}\right)^{2} \\
=4 k^{4}(1+x y-h \zeta)^{2} \\
v_{z}=-k\left(\cos \theta_{1}+\cos \theta_{2}\right) \\
=-k(x+y)
\end{gathered}
$$

$$
\begin{gathered}
v_{t}^{2}=k^{2}\left(\sin \theta_{1}^{2}+\sin \theta_{2}^{2}-2 \cos \phi_{2} \sin \theta_{1} \sin \theta_{2}\right) \\
=k^{2}\left(2-x^{2}-y^{2}-2 h \zeta\right) \\
g=\sigma^{2} v_{z}^{2}=k^{2} \sigma^{2}(x+y)^{2} .
\end{gathered}
$$

So we have

$$
\left.\frac{d P_{2}}{d \Omega_{2}}\right|_{\text {diff }}=P_{0} \frac{R s^{2} e^{-g}}{4 \pi y} \frac{(1+x y-h \zeta)^{2}}{(x+y)^{2}} \sum_{m=1}^{\infty} X_{m}
$$

in which

$$
s=k T=\frac{2 \pi T}{\lambda},
$$

where $\lambda$ is the wavelength of the radiation. We also have

$$
\begin{aligned}
X_{m} & =\frac{g^{m}}{m ! m} \mathrm{e}^{-\frac{v_{t}^{2} T^{2}}{4 m}} \\
& =\frac{g^{m}}{m ! m} \mathrm{e}^{-q s^{2} / 4 m}
\end{aligned}
$$

in which

$$
q=2-x^{2}-y^{2}-2 h \zeta .
$$

So

$\left.\frac{d P_{2}}{d \Omega_{2}}\right|_{\text {diff }}=P_{0} \frac{R s^{2}}{4 \pi y} \frac{(1+x y-h \zeta)^{2}}{(x+y)^{2}} \times \mathrm{e}^{-g} \sum_{m=1}^{\infty} \frac{g^{m}}{m ! m} \mathrm{e}^{-q s^{2} / 4 m}$.

Generally, the sum may be evaluated numerically to obtain the differential power distribution.

The parameter $s$ will be large if the correlation length is much larger than the wavelength of the radiation. Since the correlation length for a technical surface is typically $T \sim 5 \mu \mathrm{m}, s=1$ corresponds to a radiation wavelength of $31 \mu \mathrm{m}$ (photon energy of about $0.04 \mathrm{eV}$ ). The condition $s \gg 1$ for a technical surface with $T \sim 5 \mu \mathrm{m}$ thus corresponds to photon energies much larger than $0.04 \mathrm{eV}$.

If $q s^{2}$ is large, the number of terms in the sum can become very large before convergence is reached, although the total power may be very small. This can present a problem for numerical evaluation. To mitigate this, we make an estimate of the size of the sum, to decide whether to evaluate it, or simply set the power to zero, as follows. The maximum value of the summand $X_{m}$ occurs when

$$
\frac{d X_{m}}{d m}=0
$$

which implies

$$
q s^{2}-4 m_{\max }+4 m_{\max }^{2}\left(\ln g-\psi\left(m_{\max }+1\right)\right)=0
$$

in which $\psi$ is the digamma function. We can solve this equation numerically to find $m_{\max }$. Then we estimate 


$$
\sum_{m=1}^{\infty} X_{m} \lesssim m_{\max } \frac{g^{m_{\max }}}{m_{\max } ! m_{\max }} \mathrm{e}^{-\frac{q s^{2}}{4 m_{\max }}}
$$

so

$$
\ln \sum_{m=1}^{\infty} X_{m} \lesssim m_{\max } \ln g-\ln m_{\max } !-\frac{q s^{2}}{4 m_{\max }} .
$$

If this is less than, for example, -200 , we set the power to zero. Otherwise we evaluate the sum.

\section{Large s limit}

If $s \gg 1$, the argument of the exponential in the sum in Eq. (A97),

$$
\mu=-\frac{q s^{2}}{4 m}
$$

will be large, and the exponential itself will be small, unless $q$ is small. $q$ vanishes in the specular direction, when $x=y$ and $\zeta=1$. For directions close to specular, for which $x=y+\Delta x$ and $\phi_{2}=\Delta \phi$, where $\Delta x \ll 1$ and $\Delta \phi \ll 1$, we can write

$$
\begin{aligned}
\mu & =-\frac{q s^{2}}{4 m} \\
& =-s^{2} \frac{\left(2-x^{2}-y^{2}-2 h \zeta\right)}{4 m} \\
& \approx-\frac{s^{2}}{4 m}\left[\Delta x^{2} \frac{y^{2}}{1-y^{2}}+\Delta \phi^{2}\left(1-y^{2}\right)\right] .
\end{aligned}
$$

Thus, we can write the exponential in the form

$$
\mathrm{e}^{\mu} \approx \mathrm{e}^{-\frac{\Delta x^{2}}{4 \epsilon_{1}}} \mathrm{e}^{-\frac{\Delta \phi^{2}}{4 \epsilon_{2}}}
$$

in which

$$
\begin{aligned}
\epsilon_{1} & =\frac{m\left(1-y^{2}\right)}{s^{2} y^{2}} \\
\epsilon_{2} & =\frac{m}{s^{2}\left(1-y^{2}\right)} .
\end{aligned}
$$

As $s \rightarrow \infty, \epsilon_{i} \rightarrow 0$. In this limit, we can use

$$
\delta(x)=\lim _{\epsilon \rightarrow 0} \frac{1}{2 \sqrt{\pi \epsilon}} \mathrm{e}^{-x^{2} /(4 \epsilon)} .
$$

So

$$
\begin{aligned}
& \delta(\Delta x) \delta(\Delta \phi) \\
& =\left(\lim _{\epsilon_{1} \rightarrow 0} \frac{1}{2 \sqrt{\pi \epsilon_{1}}} \mathrm{e}^{-\frac{\Delta x^{2}}{4 \epsilon_{1}}}\right)\left(\lim _{\epsilon_{2} \rightarrow 0} \frac{1}{2 \sqrt{\pi \epsilon_{2}}} \mathrm{e}^{-\frac{\Delta \phi^{2}}{4 \epsilon_{2}}}\right) \\
& =\lim _{s \rightarrow \infty} \frac{s^{2} y}{4 \pi m} \mathrm{e}^{\mu} .
\end{aligned}
$$

Thus, from Eq. (A97),

$$
\begin{aligned}
\left.\lim _{s \rightarrow \infty} \frac{d P_{2}}{d \Omega_{2}}\right|_{\text {diff }}= & P_{0} \frac{R}{y^{2}} \frac{(1+x y-h \zeta)^{2}}{(x+y)^{2}} \\
& \times \mathrm{e}^{-g} \sum_{m=1}^{\infty} \frac{g^{m}}{m !} \lim _{s \rightarrow \infty} \frac{s^{2} y}{4 \pi m} \mathrm{e}^{\mu} \\
= & P_{0} \frac{R}{y^{2}} \frac{(1+x y-h \zeta)^{2}}{(x+y)^{2}} \mathrm{e}^{-g} \delta(\Delta x) \delta(\Delta \phi) \sum_{m=1}^{\infty} \frac{g^{m}}{m !} \\
= & P_{0} \frac{R}{y^{2}} \frac{(1+x y-h \zeta)^{2}}{(x+y)^{2}}\left(1-\mathrm{e}^{-g}\right) \delta(\Delta x) \delta(\Delta \phi) .
\end{aligned}
$$

Because of the delta functions which restrict the angular dependence to the specular direction, we can set $x=y$, $\zeta=1$, and $h=\left(1-y^{2}\right)$ to get

$$
\left.\lim _{s \rightarrow \infty} \frac{1}{P_{0}} \frac{d P_{2}}{d \Omega_{2}}\right|_{\text {diff }}=R\left(1-\mathrm{e}^{-g}\right) \delta(\Delta x) \delta(\Delta \phi) .
$$

The total diffuse scattering is

$\lim _{s \rightarrow \infty} P_{\text {diff }}=\left.\int d \Omega_{2} \lim _{s \rightarrow \infty} \frac{1}{P_{0}} \frac{d P_{2}}{d \Omega_{2}}\right|_{\text {diff }}=\left(1-e^{-g}\right) R$

as expected from Eq. (A79).

\section{Small g limit}

The parameter $g$ is

$$
g=(\sigma k)^{2}(x+y)^{2}=4\left(\frac{2 \pi \sigma}{\lambda}\right)^{2} \sin ^{2} \gamma
$$

in which we take the grazing angles of incidence and reflection to both be $\gamma=\pi / 2-\theta_{1}$. For small grazing angles, $g$ will be small if the rms surface roughness is much less than the wavelength of the radiation. For a technical surface, the rms surface roughness is typically $\sigma \sim 0.2 \mu \mathrm{m}$. For a grazing angle of $30 \mathrm{mrad}, g=1$ corresponds to a radiation wavelength of 0.075 microns (photon energy of $16 \mathrm{eV}$ ). Thus, at this grazing angle for a technical surface, $g \ll 1$ corresponds to photon energies much less than $16 \mathrm{eV}$.

For $g \ll 1$, we keep only the first term in the sum in Eq. (A97). Then the diffuse power is

$$
\begin{aligned}
\left.\frac{d P_{2}}{d \Omega_{2}}\right|_{\text {diff }} \approx & P_{0} \frac{R s^{2}}{4 \pi y} \frac{(1+x y-h \zeta)^{2}}{(x+y)^{2}} g \mathrm{e}^{-\left(g+q s^{2} / 4\right)} \\
\approx & P_{0} \frac{R(s k \sigma)^{2}}{4 \pi y}(1+x y-h \zeta)^{2} \\
& \times \mathrm{e}^{-(k \sigma)^{2}(x+y)^{2}} \mathrm{e}^{-\frac{\left(2-x^{2}-y^{2}-2 h \zeta\right) s^{2}}{4}} .
\end{aligned}
$$

\section{Large $g$ limit}

The opposite limit, $g \gg 1$ corresponds (for a technical surface and a $30 \mathrm{mrad}$ grazing angle) to photon energies 
much larger than $16 \mathrm{eV}$. In this case, the reflection is almost entirely diffuse. For $g \gg 1$, a power series expansion in $g$ is not useful. We return to Eq. (A56) and Eq. (A64) to obtain

$$
\begin{aligned}
\left\langle\frac{d P_{2}}{d \Omega_{2}}\right\rangle= & P_{0} \frac{R}{(4 \pi)^{2} A \cos \theta_{1}} \frac{v^{4}}{v_{z}^{2}} \\
& \times \int d \vec{\rho} d \overrightarrow{\rho^{\prime}} \mathrm{e}^{-g(1-C(r / T))} \mathrm{e}^{-l \overrightarrow{v_{t}} \cdot \vec{r}} .
\end{aligned}
$$

Considered as a function of $r$, the integrand

$$
\mathrm{e}^{-g[1-C(r / T)]}=\mathrm{e}^{-g\left(1-\mathrm{e}^{-\frac{r^{2}}{T^{2}}}\right)}
$$

falls off extremely rapidly with $r / T$ for $g \gg 1$. In this case, we can make the approximation

$$
1-\mathrm{e}^{-\frac{r^{2}}{T^{2}}} \approx \frac{r^{2}}{T^{2}}
$$

as well as extending the range of integration on $\vec{r}$ to infinity, giving

$$
\begin{aligned}
& \int d \vec{\rho} d \overrightarrow{\rho^{\prime}} \mathrm{e}^{-g[1-C(r / T)]} \mathrm{e}^{-l \overrightarrow{v_{t}} \cdot \vec{r}} \\
& \approx \int d \vec{\rho} \int_{0}^{\infty} r d r \mathrm{e}^{-\frac{g r^{2}}{T^{2}}} \int_{0}^{2 \pi} d \beta \mathrm{e}^{-l v_{t} r \cos \beta} \\
& \approx 2 \pi A \int_{0}^{\infty} r d r \mathrm{e}^{-\frac{g r^{2}}{T^{2}}} J_{0}\left(r v_{t}\right) \\
& \approx 2 \pi A \frac{T^{2}}{2 g} \mathrm{e}^{\frac{-T^{2} v_{t}^{2}}{4 g}}
\end{aligned}
$$

in which $A=\pi a^{2}$ is the area of the scattering region. Thus

$$
\begin{aligned}
\left\langle\frac{d P_{2}}{d \Omega_{2}}\right\rangle & =P_{0} \frac{R}{8 \pi \cos \theta_{1}} \frac{v^{4}}{v_{z}^{2}} \frac{T^{2}}{2 g} \mathrm{e}^{\frac{-T^{2} v_{t}^{2}}{4 g}} \\
& =P_{0} \frac{R}{2 \pi y} \frac{k^{2}(1+x y-h \zeta)^{2}}{(x+y)^{2}} \frac{T^{2}}{2 g} \mathrm{e}^{\frac{-T^{2} v_{t}^{2}}{4 g}} \\
& =P_{0} \frac{R \tau^{2}}{4 \pi y} \frac{(1+x y-h \zeta)^{2}}{(x+y)^{4}} \mathrm{e}^{-\frac{\left.\tau^{2}\left(2-x^{2}-y^{2}-2 h\right)^{2}\right)}{4(x+y)^{2}}}
\end{aligned}
$$

in which $\tau=T / \sigma$. Note that this result is independent of the radiation wavelength $\lambda$. Thus, for very large $g$ (high energy photons, except at very small grazing angles), the scattering distribution (which is essentially all diffuse) becomes energy-independent for a Gaussian autocorrelation function.

\section{Large $g$ and $\tau$ limit}

Typically $T \gg \sigma$, so $\tau \gg 1$. In this case, the differential power distribution will be close to zero except when $2-x^{2}-y^{2}-2 h \zeta \sim 0$, which is the specular direction, when $x=y$ and $\zeta=1$. For directions close to specular, for which $x=y+\Delta x$ and $\phi_{2}=\Delta \phi$, where $\Delta x \ll 1$ and $\Delta \phi \ll 1$, we can write

$$
\begin{aligned}
\mu^{\prime} & =-\frac{\tau^{2}\left(2-x^{2}-y^{2}-2 h \zeta\right)}{4(x+y)^{2}} \\
& \approx-\frac{\tau^{2}}{16}\left(\Delta x^{2} \frac{1}{1-y^{2}}+\Delta \phi^{2} \frac{1-y^{2}}{y^{2}}\right) .
\end{aligned}
$$

As above, we can write the exponential in the form

$$
\mathrm{e}^{\mu^{\prime}} \approx \mathrm{e}^{-\frac{\Delta x^{2}}{4 \epsilon_{1}}} \mathrm{e}^{-\frac{\Delta \phi^{2}}{4 \epsilon_{2}}}
$$

in which

$$
\begin{gathered}
\epsilon_{1}=\frac{4\left(1-y^{2}\right)}{\tau^{2}} \\
\epsilon_{2}=\frac{4 y^{2}}{1-y^{2}} .
\end{gathered}
$$

So

$$
\begin{aligned}
& \delta(\Delta x) \delta(\Delta \phi) \\
& =\left(\lim _{\epsilon_{1} \rightarrow 0} \frac{1}{2 \sqrt{\pi \epsilon_{1}}} \mathrm{e}^{-\frac{\Delta x^{2}}{4 \epsilon_{1}}}\right)\left(\lim _{\epsilon_{2} \rightarrow 0} \frac{1}{2 \sqrt{\pi \epsilon_{2}}} \mathrm{e}^{-\frac{\Delta \phi^{2}}{4 \epsilon_{2}}}\right) \\
& =\lim _{\tau \rightarrow \infty} \frac{\tau^{2}}{16 \pi y} \mathrm{e}^{\mu^{\prime}} .
\end{aligned}
$$

Thus, from Eq. (A111),

$$
\begin{aligned}
\lim _{\tau \rightarrow \infty} & \left\langle\frac{d P_{2}}{d \Omega_{2}}\right\rangle \\
& =P_{0} \frac{4 R(1+x y-h \zeta)^{2}}{(x+y)^{4}}\left(\lim _{s \rightarrow \infty} \frac{\tau^{2}}{16 \pi y} \mathrm{e}^{\mu^{\prime}}\right) \\
& =P_{0} \frac{4 R(1+x y-h \zeta)^{2}}{(x+y)^{4}} \delta(\Delta x) \delta(\Delta \phi) .
\end{aligned}
$$

Because of the delta functions which restrict the angular dependence to the specular direction, we can set $x=y$, $\zeta=1$, and $h=\left(1-y^{2}\right)$ to get

$$
\lim _{\tau \rightarrow \infty} \frac{1}{P_{0}}\left\langle\frac{d P_{2}}{d \Omega_{2}}\right\rangle=R \delta(\Delta x) \delta(\Delta \phi) .
$$

The total scattering, which is all diffuse but also all in the specular direction, is

$$
\lim _{\tau \rightarrow \infty} P_{\text {scatt }}=\int d \Omega_{2} \lim _{\tau \rightarrow \infty} \frac{1}{P_{0}}\left\langle\frac{d P_{2}}{d \Omega_{2}}\right\rangle=R
$$

as expected from Eq. (A79). 


\section{d. Diffuse power evaluation: Exponential autocorrelation function}

For an exponential autocorrelation function, we have

$$
C(r / T)=\mathrm{e}^{-\frac{r}{T}}
$$

so the diffuse power, expressed in terms of a power series from Eq. (A76), is

$\left.\frac{d P_{2}}{d \Omega_{2}}\right|_{\text {diff }}=P_{0} \frac{R e^{-g}}{8 \pi \cos \theta_{1}} \frac{v^{4}}{v_{z}^{2}} \times \sum_{m=1}^{\infty} \int_{0}^{\infty} r d r \frac{g^{m} \mathrm{e}^{-\frac{m r}{T}}}{m !} J_{0}\left(r v_{t}\right)$.

The integral is

$$
\int_{0}^{\infty} r d r \mathrm{e}^{-\frac{m r}{T}} J_{0}\left(r v_{t}\right)=\frac{T^{2}}{m^{2}\left(1+\frac{T^{2} v_{t}^{2}}{m^{2}}\right)^{3 / 2}}
$$

so the power is

$$
\begin{aligned}
\left.\frac{d P_{2}}{d \Omega_{2}}\right|_{\text {diff }}= & P_{0} \frac{R}{8 \pi \cos \theta_{1}} \frac{v^{4} T^{2}}{v_{z}^{2}} \\
& \times e^{-g} \sum_{m=1}^{\infty} \frac{g^{m}}{m ! m^{2}\left(1+\frac{T^{2} v_{t}^{2}}{m^{2}}\right)^{3 / 2}} \\
= & P_{0} \frac{R}{2 \pi y} \frac{s^{2}(1+x y-h \zeta)^{2}}{(x+y)^{2}} \\
& \times e^{-g} \sum_{m=1}^{\infty} \frac{g^{m}}{m ! m^{2}\left[1+\frac{s^{2}\left(2-x^{2}-y^{2}-2 h \zeta\right)}{m^{2}}\right]^{3 / 2}} .
\end{aligned}
$$

\section{Large s limit}

If $s \gg 1$, the summand will be small unless $2-x^{2}-$ $y^{2}-2 h \zeta$ is small. This quantity vanishes in the specular direction, when $x=y$ and $\zeta=1$. For directions close to specular, for which $x=y+\Delta x$ and $\phi_{2}=\Delta \phi$, where $\Delta x \ll 1$ and $\Delta \phi \ll 1$, we can write

$$
\frac{s^{2}\left(2-x^{2}-y^{2}-2 h \zeta\right)}{m^{2}} \approx \frac{s^{2}}{m^{2}}\left[\Delta x^{2} \frac{y^{2}}{1-y^{2}}+\Delta \phi^{2}\left(1-y^{2}\right)\right]
$$

so

$$
\begin{aligned}
& \frac{m^{2}}{s^{2}}\left[1+\frac{s^{2}\left(2-x^{2}-y^{2}-2 h \zeta\right)}{m^{2}}\right]^{3 / 2} \\
& \quad \approx \frac{s}{m}\left[\frac{m^{2}}{s^{2}}+\Delta x^{2} \frac{y^{2}}{1-y^{2}}+\Delta \phi^{2}\left(1-y^{2}\right)\right]^{3 / 2} .
\end{aligned}
$$

Thus, we can write

$$
\begin{aligned}
& \frac{m^{2}}{s^{2}}\left[1+\frac{s^{2}\left(2-x^{2}-y^{2}-2 h \zeta\right)}{m^{2}}\right]^{3 / 2} \\
& \quad \approx \frac{1}{\epsilon}\left(\epsilon^{2}+\kappa_{1} \Delta x^{2}+\kappa_{2} \Delta \phi^{2}\right)^{3 / 2}
\end{aligned}
$$

in which

$$
\begin{gathered}
\epsilon=\frac{m}{s} \\
\kappa_{1}=\frac{y^{2}}{1-y^{2}} \\
\kappa_{2}=1-y^{2} .
\end{gathered}
$$

As $s \rightarrow \infty, \epsilon \rightarrow 0$. In this limit, we can use

$$
\delta(x) \delta(y)=\lim _{\epsilon \rightarrow 0} \frac{\epsilon \sqrt{\kappa_{1} \kappa_{2}}}{2 \pi\left(\epsilon^{2}+\kappa_{1} x^{2}+\kappa_{2} y^{2}\right)^{3 / 2}} .
$$

So

$$
\begin{aligned}
& \delta(\Delta x) \delta(\Delta \phi) \\
& =\lim _{\epsilon \rightarrow 0} \frac{\epsilon \sqrt{\kappa_{1} \kappa_{2}}}{2 \pi\left(\epsilon^{2}+\kappa_{1} \Delta x^{2}+\kappa_{2} \Delta \phi^{2}\right)^{3 / 2}} \\
& =\lim _{s \rightarrow \infty} \frac{y}{2 \pi} \frac{s^{2}}{m^{2}}\left[1+\frac{s^{2}\left(2-x^{2}-y^{2}-2 h \zeta\right)}{m^{2}}\right]^{-3 / 2}
\end{aligned}
$$

Thus, from Eq. (A122),

$$
\begin{aligned}
\left.\lim _{s \rightarrow \infty} \frac{d P_{2}}{d \Omega_{2}}\right|_{\text {diff }} \\
=P_{0} \frac{R}{y^{2}} \frac{(1+x y-h \zeta)^{2}}{(x+y)^{2}} \\
\quad \times e^{-g} \sum_{m=1}^{\infty} \frac{g^{m}}{m !} \lim _{s \rightarrow \infty} \frac{y s^{2}}{2 \pi m^{2}\left[1+\frac{s^{2}\left(2-x^{2}-y^{2}-2 h \zeta\right)}{m^{2}}\right]^{3 / 2}} \\
=P_{0} \frac{R}{y^{2}} \frac{(1+x y-h \zeta)^{2}}{(x+y)^{2}} \mathrm{e}^{-g} \delta(\Delta x) \delta(\Delta \phi) \sum_{m=1}^{\infty} \frac{g^{m}}{m !} \\
=P_{0} \frac{R}{y^{2}} \frac{(1+x y-h \zeta)^{2}}{(x+y)^{2}}\left(1-\mathrm{e}^{-g}\right) \delta(\Delta x) \delta(\Delta \phi)
\end{aligned}
$$

Because of the delta functions which restrict the angular dependence to the specular direction, we can set $x=y$, $\zeta=1$, and $h=\left(1-y^{2}\right)$ to get

$\left.\lim _{s \rightarrow \infty} \frac{1}{P_{0}} \frac{d P_{2}}{d \Omega_{2}}\right|_{\text {diff }}=R\left(1-\mathrm{e}^{-g(x, y)}\right) \delta(\Delta x) \delta(\Delta \phi)$.

The total diffuse scattering is 
$\lim _{s \rightarrow \infty} P_{\text {diff }}=\left.\int d \Omega_{2} \lim _{s \rightarrow \infty} \frac{1}{P_{0}} \frac{d P_{2}}{d \Omega_{2}}\right|_{\text {diff }}=\left(1-e^{-g}\right) R$

as expected from Eq. (A79).

\section{Large g limit}

For $g \gg 1$, the power series expansion in $g$ is not useful. Again, we return to Eq. (A56) and Eq. (A64) to obtain

$\left\langle\frac{d P_{2}}{d \Omega_{2}}\right\rangle=P_{0} \frac{R}{(4 \pi)^{2} A \cos \theta_{1}} \frac{v^{4}}{v_{z}^{2}} \int d \vec{\rho} d \overrightarrow{\rho^{\prime}} \mathrm{e}^{-g[1-C(r / T)]} \mathrm{e}^{-l \overrightarrow{v_{t}} \cdot \vec{r}}$.

Considered as a function of $r$, the integrand

$$
\mathrm{e}^{-g[1-C(r / T)]}=\mathrm{e}^{-g\left(1-\mathrm{e}^{-\frac{r}{T}}\right)}
$$

falls off extremely rapidly with $r / T$ for $g \gg 1$. In this case, we can make the approximation

$$
1-\mathrm{e}^{-\frac{r}{T}} \approx \frac{r}{T}
$$

as well as extending the range of integration on $\vec{r}$ to infinity, giving

$$
\begin{aligned}
& \int d \vec{\rho} d \overrightarrow{\rho^{\prime}} \mathrm{e}^{-g[1-C(r / T)]} \mathrm{e}^{-l \vec{v}_{t} \cdot \vec{r}} \\
& \approx \int d \vec{\rho} \int_{0}^{\infty} r d r \mathrm{e}^{-\frac{g r}{T}} \int_{0}^{2 \pi} d \beta \mathrm{e}^{-l v_{t} r \cos \beta} \\
& \approx 2 \pi A \int_{0}^{\infty} r d r \mathrm{e}^{-\frac{g r}{T}} J_{0}\left(r v_{t}\right) \\
& \approx 2 \pi A \frac{T^{2}}{g^{2}\left(1+\frac{T^{2} v_{t}^{2}}{g^{2}}\right)^{3 / 2}}
\end{aligned}
$$

in which $A=\pi a^{2}$ is the area of the scattering region. Thus

$$
\begin{aligned}
\left\langle\frac{d P_{2}}{d \Omega_{2}}\right\rangle & =P_{0} \frac{R}{8 \pi \cos \theta_{1}} \frac{v^{4}}{v_{z}^{2}} \frac{T^{2}}{g^{2}\left(1+\frac{T^{2} v_{t}^{2}}{g^{2}}\right)^{3 / 2}} \\
& =P_{0} \frac{R}{2 \pi y} \frac{\tau^{4}(1+x y-h \zeta)^{2}}{s^{2}(x+y)^{6}} \frac{1}{\left[1+\frac{\tau^{4}\left(2-x^{2}-y^{2}-2 h \zeta\right)}{(x+y)^{4} s^{2}}\right]^{3 / 2}} .
\end{aligned}
$$

Unlike the case of a Gaussian autocorrelation, this result is not independent of the radiation wavelength $\lambda$.

\section{Probability distributions}

\section{a. General definitions}

The differential angular power distribution, normalized to the total power, may be interpreted as the joint probability distribution of a photon scattering at a polar angle $\theta_{2}$ and azimuthal angle $\phi_{2}=\phi$. (In this section, the azimuthal scattering angle $\phi_{2}$ will be designated as simply $\phi$.) Thus, the diffuse scattering joint probability distribution is

$$
P(x, \phi)=\left.\frac{1}{N_{0}} \frac{1}{P_{0}} \frac{d P_{2}}{d \Omega_{2}}\right|_{\text {diff }} .
$$

The normalization factor $N_{0}$ is

$$
N_{0}^{-1}=\left.\int_{0}^{2 \pi} d \phi \int_{-1}^{1} d x \frac{1}{P_{0}} \frac{d P_{2}}{d \Omega_{2}}\right|_{\text {diff }}
$$

The marginal probability distribution in $x$ is

$$
P_{x}(x)=\int_{0}^{2 \pi} d \phi P(x, \phi) .
$$

This is the probability distribution in $x$, irrespective of $\phi$. The marginal probability distribution in $\phi$ is

$$
P_{\phi}(\phi)=\int_{-1}^{1} d x P(x, \phi) .
$$

This is the probability distribution in $\phi$, irrespective of $x$. The conditional probability in $x$ is

$$
P(x \mid \phi)=\frac{P(x, \phi)}{P_{\phi}(\phi)} .
$$

This is the probability that, given a specific value of $\phi, x$ occurs. The conditional probability in $\phi$ is

$$
P(\phi \mid x)=\frac{P(x, \phi)}{P_{x}(x)} .
$$

This is the probability that, given a specific value of $x, \phi$ occurs.

\section{b. Methodology for generating samples for $\boldsymbol{x}$ and $\phi$}

To generate the scattering parameters $x$ and $\phi$, sampled from the joint probability distribution $P(x, \phi)$, we first sample from the marginal probability distribution in $x$. To do this efficiently, we form the marginal cumulative distribution function in $x$ :

$$
C_{x}(x)=\int_{-1}^{x} d x^{\prime} P_{x}\left(x^{\prime}\right) .
$$

The form of $P_{x}(x)$ does not admit to doing the integral analytically. As an efficient alternative to direct numerical integration, we first expand the function as a series of $N$ orthogonal Chebyshev polynomials $T_{n}(x)$ : 


$$
P_{x}(x)=\frac{c_{0}}{2}+\sum_{n=1}^{n=N} c_{n} T_{n}(x)
$$

in which

$$
T_{n}(x)=\cos \left(n \cos ^{-1} x\right)
$$

and

$$
c_{n}=\frac{2}{\pi} \int_{-1}^{1} d x \frac{T_{n}(x) P_{x}(x)}{\sqrt{1-x^{2}}} .
$$

Typically, the distribution $P_{x}(x)$ is peaked near $y=\cos \theta_{1}$ and the expansion requires only a modest number $N$ of terms in the series for adequate convergence. The integrals of Chebyshev polynomials can be done analytically:

$$
\int_{-1}^{x} d x^{\prime} T_{n}\left(x^{\prime}\right)=S_{n}(x)
$$

in which

$$
\begin{aligned}
\left(1-n^{2}\right) S_{n}(x)= & \cos n \pi+x \cos \left(n \cos ^{-1} x\right) \\
& +n \sqrt{1-x^{2}} \sin \left(n \cos ^{-1} x\right)
\end{aligned}
$$

so

$$
C_{x}(x)=\frac{c_{0} S_{0}(x)}{2}+\sum_{n=1}^{n=N} c_{n} S_{n}(x) .
$$

To find a sample value $x$, we choose a random number $R_{1}$ between 0 and 1 , set $R_{1}=C_{x}(x)$, and solve for $x$. This may be done numerically, for example using the NewtonRaphson method. Then, given this value of $x$, the conditional probability distribution in $\phi$ is

$C_{\phi}(x, \phi)=\int_{0}^{\phi} d \phi^{\prime} P\left(\phi^{\prime} \mid x\right)=\frac{1}{P_{x}(x)} \int_{0}^{\phi} d \phi^{\prime} P\left(x, \phi^{\prime}\right)$.

As discussed below, this integral may be done analytically. Then, we choose a second random number $R_{2}$ between 0 and 1 , set $R_{2}=C_{\phi}(\phi)$ and find $\phi$, again using the NewtonRaphson method.

\section{c. Gaussian autocorrelation: Probability distributions}

For a Gaussian autocorrelation function, the diffuse scattering joint probability distribution is, from Eq. (A97),

$$
\begin{aligned}
P(x, \phi)= & \frac{R s^{2}}{4 N_{0} \pi y} \frac{(1+x y-h \zeta)^{2}}{(x+y)^{2}} \\
& \times \mathrm{e}^{-g} \sum_{m=1}^{\infty} \frac{g^{m}}{m ! m} \mathrm{e}^{-q s^{2} / 4 m}
\end{aligned}
$$

in which $\zeta=\cos \phi$ and $q=2-x^{2}-y^{2}-2 h \zeta$. To separate out the $\phi$ dependence, we define

$$
\begin{gathered}
\alpha=\frac{h}{1+x y} \\
\beta=\frac{h s^{2}}{2} .
\end{gathered}
$$

Also, if we let $\nu=k \sigma$, then

$$
g=\nu^{2}(x+y)^{2}
$$

so

$$
\begin{aligned}
P(x, \phi)= & \frac{R s^{2}}{4 N_{0} \pi y} \frac{(1+x y)^{2}(1-\alpha \cos \phi)^{2}}{(x+y)^{2}} \mathrm{e}^{-\nu^{2}(x+y)^{2}} \\
& \times \sum_{m=1}^{\infty} \frac{\nu^{2 m}(x+y)^{2 m}}{m ! m} \mathrm{e}^{-\frac{\left(2-x^{2}-y^{2}\right) s^{2}}{4 m}} \mathrm{e}^{\frac{\beta \cos \phi}{m}} .
\end{aligned}
$$

\section{Marginal probability distribution in $x$}

From Eq. (A141),

$$
\begin{aligned}
P_{x}(x) & \\
= & \int_{0}^{2 \pi} d \phi P(x, \phi) \\
= & \frac{R s^{2}}{4 N_{0} \pi y} \frac{(1+x y)^{2}}{(x+y)^{2}} \mathrm{e}^{-\nu^{2}(x+y)^{2}} \sum_{m=1}^{\infty} \frac{\nu^{2 m}(x+y)^{2 m}}{m ! m} \\
& \times \mathrm{e}^{-\frac{\left(2-x^{2}-y^{2}\right) s^{2}}{4 m}} \int_{0}^{2 \pi} d \phi(1-\alpha \cos \phi)^{2} \mathrm{e}^{\frac{\beta \cos \phi}{m}} .
\end{aligned}
$$

To do the integral, we use

$$
\mathrm{e}^{z \cos \phi}=I_{0}(z)+2 \sum_{k=1}^{\infty} I_{k}(z) \cos k \phi .
$$

Then we have

$$
\int_{0}^{\phi} d \phi^{\prime}\left(1-\alpha \cos \phi^{\prime}\right)^{2} \mathrm{e}^{\beta \cos \phi^{\prime}}=2 \pi S_{I}(\beta, \phi)
$$

in which

$$
\begin{aligned}
2 \pi S_{I}(\beta, \phi)= & r_{0}(\alpha, \phi) I_{0}(\beta)+2 r_{1}(\alpha, \phi) I_{1}(\beta) \\
& +2 r_{2}(\alpha, \phi) I_{2}(\beta)+2 \sum_{k=3}^{\infty} r_{k}(\alpha, \phi) I_{k}(\beta)
\end{aligned}
$$

and

$$
r_{0}(\alpha, \phi)=\left(1+\alpha^{2} / 2\right) \phi-2 \alpha \sin \phi+\left(\alpha^{2} / 4\right) \sin 2 \phi
$$




$$
\begin{aligned}
r_{1}(\alpha, \phi)= & -\alpha \phi+\left(1+3 \alpha^{2} / 4\right) \sin \phi \\
& -\alpha \cos \phi \sin \phi+\left(\alpha^{2} / 12\right) \sin 3 \phi
\end{aligned}
$$

$$
\begin{aligned}
r_{2}(\alpha, \phi)= & \left(\alpha^{2} / 4\right) \phi-\alpha \sin \phi \\
& +\frac{12\left(2+\alpha^{2}\right) \sin 2 \phi-16 \alpha \sin 3 \phi+3 \alpha^{2} \sin 4 \phi}{48}
\end{aligned}
$$

$$
\begin{aligned}
r_{k}(\alpha, \phi)= & \frac{2 \alpha \cos k \phi \sin \phi}{k^{2}-1} \\
& -2 \alpha \cos \phi\left(\frac{\alpha \cos k \phi \sin \phi}{k^{2}-4}+\frac{k \sin k \phi}{k^{2}-1}\right) \\
& +\sin k \phi \frac{\left(2+\alpha^{2}\right)\left(k^{2}-4\right)+\alpha^{2} k^{2} \cos 2 \phi}{2 k\left(k^{2}-4\right)}
\end{aligned}
$$

For $\phi=2 \pi$

$$
\begin{aligned}
& r_{0}(\alpha, 2 \pi)=\left(1+\alpha^{2} / 2\right) 2 \pi \\
& r_{1}(\alpha, 2 \pi)=-2 \alpha \pi \\
& r_{2}(\alpha, 2 \pi)=\left(\alpha^{2} / 2\right) \pi \\
& r_{k}(\alpha, 2 \pi)=0
\end{aligned}
$$

so

$$
\begin{aligned}
2 \pi & S_{I}(\beta, 2 \pi) \\
& =2 \pi\left[\left(1+\alpha^{2} / 2\right) I_{0}(\beta)-2 \alpha I_{1}(\beta)+\frac{\alpha^{2}}{2} I_{2}(\beta)\right] \\
& =2 \pi\left[\left(1+\alpha^{2}\right) I_{0}(\beta)-\frac{\alpha(\alpha+2 \beta) I_{1}(\beta)}{\beta}\right]
\end{aligned}
$$

and

$$
\int_{0}^{2 \pi} d \phi(1-\alpha \cos \phi)^{2} \mathrm{e}^{\frac{\beta \cos \phi}{m}}=2 \pi S_{I}\left(\frac{\beta}{m}, 2 \pi\right) .
$$

Thus

$$
P_{x}(x)=\frac{R s^{2}}{2 N_{0} y} \frac{(1+x y)^{2}}{(x+y)^{2}} \mathrm{e}^{-\nu^{2}(x+y)^{2}} \sum_{m=1}^{\infty} F_{m}(x)
$$

in which

$$
F_{m}(x)=\frac{\nu^{2 m}(x+y)^{2 m}}{m ! m} \mathrm{e}^{-\frac{\left(2-x^{2}-y^{2}\right) s^{2}}{4 m}} S_{I}\left(\frac{\beta}{m}, 2 \pi\right) .
$$

For $x=1$ or $y=1, \quad h=0 \quad$ so $\alpha=\beta=0$, and $S_{I}(0,2 \pi)=1$, so

$$
F_{m}(x)=\frac{\nu^{2 m}(x+y)^{2 m}}{m ! m} \mathrm{e}^{-\frac{\left(2-x^{2}-y^{2}\right) s^{2}}{4 m}}
$$

If $s \gg 1$, then $\beta \gg 1$, except near $h=0$. Direct numerical evaluation of the Bessel function for very large arguments can cause numerical problems. In this case, it is convenient to introduce the asymptotic form [25] for the Bessel function, useful for $x \gg 1$ :

$$
I_{k}(x) \cong \frac{\mathrm{e}^{x}}{\sqrt{2 \pi x}} Z_{k}(x)
$$

in which $Z_{k}(x)$ is a polynomial in inverse powers of $x$ :

$$
\begin{aligned}
Z_{k}(x)= & 1-\frac{4 k^{2}-1}{8 x}+\frac{\left(4 k^{2}-1\right)\left(4 k^{2}-9\right)}{2 !(8 x)^{2}} \\
& -\frac{\left(4 k^{2}-1\right)\left(4 k^{2}-9\right)\left(4 k^{2}-25\right)}{3 !(8 x)^{3}}+\cdots
\end{aligned}
$$

Then we can introduce $S_{Z}(\beta, \phi)$ :

$$
\begin{aligned}
S_{Z}(\beta, \phi)= & r_{0}(\alpha, \phi) Z_{0}(\beta)+2 r_{1}(\alpha, \phi) Z_{1}(\beta) \\
& +2 r_{2}(\alpha, \phi) Z_{2}(\beta)+2 \sum_{k=3}^{\infty} r_{k}(\alpha, \phi) Z_{k}(\beta)
\end{aligned}
$$

and we have

$$
S_{I}(\beta, \phi) \cong \frac{\mathrm{e}^{\beta}}{\sqrt{2 \pi \beta}} S_{Z}(\beta, \phi) .
$$

For example, for $\beta \gg 1$, to leading order in $\frac{1}{\beta}$,

$$
\begin{gathered}
\int_{0}^{2 \pi} d \phi^{\prime}\left(1-\alpha \cos \phi^{\prime}\right)^{2} \mathrm{e}^{\beta \cos \phi^{\prime}}=2 \pi S_{I}(\beta, 2 \pi) \\
\cong 2 \pi \frac{\mathrm{e}^{\beta}}{\sqrt{2 \pi \beta}} S_{Z}(\beta, 2 \pi) \cong \mathrm{e}^{\beta}(1-\alpha)^{2} \sqrt{\frac{2 \pi}{\beta}}
\end{gathered}
$$

If we return to Eq. (A165) and let

$$
q_{0}=2-x^{2}-y^{2}-2 h
$$

then, for large $\beta / m$, we can use

$$
F_{m}(x) \cong \frac{\nu^{2 m}(x+y)^{2 m}}{m ! m \sqrt{2 \pi m \beta}} \mathrm{e}^{-\frac{q_{0} s^{2}}{4 m}} S_{Z}\left(\frac{\beta}{m}, 2 \pi\right)
$$

Small g limit.- For $g \ll 1$, we keep only the first term in the power series. Thus

$$
P_{x}(x) \approx \frac{R s^{2}}{2 N_{0} y} \frac{(1+x y)^{2}}{(x+y)^{2}} \mathrm{e}^{-\nu^{2}(x+y)^{2}} F_{1}(x),
$$

with 


$$
F_{1}(x)=\nu^{2}(x+y)^{2} \mathrm{e}^{-\frac{\left(2-x^{2}-y^{2}\right) s^{2}}{4}} S_{I}(\beta, 2 \pi) .
$$

Large $g$ limit.- For $g \gg 1$, from Eq. (A111)

$$
\begin{aligned}
P(x, \phi) \approx & \frac{R \tau^{2}}{4 N_{0} \pi y} \frac{(1+x y)^{2}(1-\alpha \cos \phi)^{2}}{(x+y)^{4}} \\
& \times \mathrm{e}^{-\frac{\tau^{2}\left(2-x^{2}-y^{2}\right)}{4(x+y)^{2}}} \mathrm{e}^{\beta^{\prime} \cos \phi}
\end{aligned}
$$

in which

$$
\beta^{\prime}=\frac{h \tau^{2}}{2(x+y)^{2}}
$$

So

$$
\begin{aligned}
P_{x}(x)= & \int_{0}^{2 \pi} d \phi P(x, \phi) \\
\approx & \frac{R \tau^{2}}{4 \pi N_{0} y} \frac{(1+x y)^{2}}{(x+y)^{4}} \mathrm{e}^{-\frac{\left(2-x^{2}-y^{2}\right)^{2}}{4(x+y)^{2}}} \\
& \times \int_{0}^{2 \pi} d \phi(1-\alpha \cos \phi)^{2} e^{\beta^{\prime} \cos \phi} .
\end{aligned}
$$

Then, from Eq. (A156) and Eq. (A170)

$$
\begin{aligned}
\int_{0}^{2 \pi} d \phi^{\prime}\left(1-\alpha \cos \phi^{\prime}\right)^{2} \mathrm{e}^{\beta^{\prime} \cos \phi^{\prime}} & =2 \pi S_{I}\left(\beta^{\prime}, 2 \pi\right) \\
& \approx \frac{2 \pi \mathrm{e}^{\beta^{\prime}}}{\sqrt{2 \pi \beta^{\prime}}} S_{Z}\left(\beta^{\prime}, 2 \pi\right) .
\end{aligned}
$$

So

$$
P_{x}(x) \approx \frac{R \tau^{2}}{2 N_{0} y} \frac{(1+x y)^{2}}{(x+y)^{4}} F_{0}(x)
$$

in which

$$
\begin{aligned}
F_{0}(x) & =\mathrm{e}^{-\frac{\left(2-x^{2}-y^{2}\right) \tau^{2}}{4(x+y)^{2}}} S_{I}\left(\beta^{\prime}, 2 \pi\right) \\
& \cong \frac{\mathrm{e}^{-\frac{q_{0} \tau^{2}}{4(x+y)^{2}}}}{\sqrt{2 \pi \beta^{\prime}}} S_{Z}\left(\beta^{\prime}, 2 \pi\right) .
\end{aligned}
$$

For $x=1 \quad$ or $y=1, \quad h=0 \quad$ so $\quad \alpha=\beta^{\prime}=0$, and $S_{I}(0,2 \pi)=1$, so

$$
F_{0}(x)=\mathrm{e}^{-\frac{\left(2-x^{2}-y^{2}\right)^{2}}{4(x+y)^{2}}}
$$

\section{Cumulative probability distribution in $\phi$}

From Eq. (A149)

$$
C_{\phi}(x, \phi)=\frac{1}{P_{x}(x)} \int_{0}^{\phi} d \phi^{\prime} P\left(x, \phi^{\prime}\right),
$$

and from Eq. (A153), we have

$$
\begin{aligned}
P(x, \phi)= & \frac{R s^{2}}{4 N_{0} \pi y} \frac{(1+x y)^{2}(1-\alpha \cos \phi)^{2}}{(x+y)^{2}} \mathrm{e}^{-\nu^{2}(x+y)^{2}} \\
& \times \sum_{m=1}^{\infty} \frac{\nu^{2 m}(x+y)^{2 m}}{m ! m} \mathrm{e}^{-\frac{\left(2-x^{2}-y^{2}\right) s^{2}}{4 m}} \mathrm{e}^{\frac{\beta \cos \phi}{m}} .
\end{aligned}
$$

So

$$
C_{\phi}(x, \phi)=\frac{R s^{2}}{2 N_{0} y P_{x}(x)} \frac{(1+x y)^{2}}{(x+y)^{2}} \mathrm{e}^{-\nu^{2}(x+y)^{2}} \sum_{m=1}^{\infty} G_{m}(x, \phi)
$$

in which

$$
\begin{aligned}
G_{m}(x, \phi)= & \frac{\nu^{2 m}(x+y)^{2 m}}{2 \pi m ! m} \mathrm{e}^{-\frac{\left(2-x^{2}-y^{2}\right) s^{2}}{4 m}} \\
& \times \int_{0}^{\phi} d \phi^{\prime}\left(1-\alpha \cos \phi^{\prime}\right)^{2} \mathrm{e}^{\frac{\beta \cos \phi^{\prime}}{m}} .
\end{aligned}
$$

Using Eq. (A156) and Eq. (A170) we have

$$
\begin{aligned}
& G_{m}(x, \phi) \\
& \quad=\frac{\nu^{2 m}(x+y)^{2 m}}{m ! m} \mathrm{e}^{-\frac{\left(2-x^{2}-y^{2}\right) s^{2}}{4 m}} S_{I}(\beta / m, \phi) \\
& \cong \frac{\nu^{2 m}(x+y)^{2 m}}{m ! m \sqrt{2 \pi m \beta}} \mathrm{e}^{-\frac{q 0^{2} s^{2}}{4 m}} S_{Z}(\beta / m, \phi) .
\end{aligned}
$$

For $x=1$ or $y=1, h=0$ so $\alpha=\beta=0$, and $S_{I}(0, \phi)=\frac{\phi}{2 \pi}$, so

$$
G_{m}(x, \phi)=\frac{\nu^{2 m}(x+y)^{2 m}}{2 \pi m ! m} \mathrm{e}^{-\frac{\left(2-x^{2}-y^{2}\right) s^{2}}{4 m}} \phi .
$$

Small g limit.—For $g \ll 1$, we keep only the first term in the power series in $m$. So

$$
C_{\phi}(x, \phi) \approx \frac{R s^{2}}{4 N_{0} \pi y P_{x}(x)} \frac{(1+x y)^{2}}{(x+y)^{2}} \mathrm{e}^{-\nu^{2}(x+y)^{2}} G_{1}(x, \phi)
$$

in which

$$
G_{1}(x, \phi)=\nu^{2}(x+y)^{2} \mathrm{e}^{-\frac{\left(2-x^{2}-y^{2}\right) s^{2}}{4}} S_{I}(\beta, \phi) .
$$

Large $g$ limit._- For $g \gg 1$, from Eq. (A111), we have

$$
\begin{aligned}
P(x, \phi) \approx & \frac{R \tau^{2}}{4 N_{0} \pi y} \frac{(1+x y)^{2}(1-\alpha \cos \phi)^{2}}{(x+y)^{4}} \\
& \times \mathrm{e}^{-\frac{\tau^{2}\left(2-x^{2}-y^{2}\right)}{4(x+y)^{2}}} \mathrm{e}^{\beta^{\prime} \cos \phi}
\end{aligned}
$$

so 


$$
C_{\phi}(x, \phi)=\frac{R \tau^{2}}{2 N_{0} y P_{x}(x)} \frac{(1+x y)^{2}}{(x+y)^{4}} G_{0}(x, \phi)
$$

with

$$
\begin{aligned}
G_{0}(x, \phi) & =\frac{\mathrm{e}^{-\frac{\tau^{2}\left(2-x^{2}-y^{2}\right)}{4(x+y)^{2}}}}{2 \pi} \int_{0}^{\phi} d \phi^{\prime}\left(1-\alpha \cos \phi^{\prime}\right)^{2} \mathrm{e}^{\beta^{\prime} \cos \phi^{\prime}} \\
& =\mathrm{e}^{-\frac{\tau^{2}\left(2-x^{2}-y^{2}\right)}{4(x+y)^{2}}} S_{I}\left(\beta^{\prime}, \phi\right) \\
& \cong \frac{1}{\sqrt{2 \pi \beta^{\prime}}} \mathrm{e}^{-\frac{\tau^{2} q_{0}}{4(x+y)^{2}}} S_{Z}\left(\beta^{\prime}, \phi\right)
\end{aligned}
$$

in which the last line is useful for large $\beta^{\prime}$. For $x=1$ or $y=1, h=0$ so $\alpha=\beta^{\prime}=0$, and $S_{I}(0, \phi)=\frac{\phi}{2 \pi}$, so

$$
G_{0}(x, \phi)=\mathrm{e}^{-\tau^{2 \frac{\left(2-x^{2}-y^{2}\right)}{4(x+y)^{2}}} \frac{\phi}{2 \pi} .}
$$

Alternate evaluation of the integral over $\phi$, for very large B.- The expression presented in Eq. (A156) for the integral over $\phi$ as an infinite series does not work very well for large values of $\beta$ : the series does not converge very fast. For large $\beta$, however, the integrand is sharply peaked near $\phi=0$, so a small angle approximation can be used:

$$
\begin{aligned}
& \int_{0}^{\phi} d \phi^{\prime}\left(1-\alpha \cos \phi^{\prime}\right)^{2} \mathrm{e}^{\beta \cos \phi^{\prime}} \\
& =\mathrm{e}^{\beta} \int_{0}^{\phi} d \phi^{\prime}\left(1-\alpha \cos \phi^{\prime}\right)^{2} \mathrm{e}^{-\beta\left(1-\cos \phi^{\prime}\right)} \\
& \approx \mathrm{e}^{\beta} \int_{0}^{\phi} d \phi^{\prime}\left(1-\alpha+\alpha \phi^{\prime 2} / 2\right)^{2} \mathrm{e}^{-\beta \phi^{\prime 2} / 2} .
\end{aligned}
$$

Define

$$
\begin{aligned}
H(\beta, \phi)= & \frac{1}{2 \pi} \int_{0}^{\phi} d \phi^{\prime}\left(1-\alpha+\alpha \phi^{\prime 2} / 2\right)^{2} \mathrm{e}^{-\beta \phi^{\prime 2} / 2} \\
= & -\frac{\alpha \phi \mathrm{e}^{-\beta \phi^{2} / 2}\left[4 b+\alpha\left(3+\beta\left(\phi^{2}-4\right)\right]\right.}{8 \pi \beta^{2}} \\
& +\sqrt{\frac{\pi}{2}} \operatorname{erf}\left(\phi \sqrt{\frac{\beta}{2}}\right) \\
& \times \frac{4 \alpha(1-2 \beta) \beta+4 \beta^{2}+\alpha^{2}\left(3-4 \beta+4 \beta^{2}\right)}{8 \pi \beta^{5 / 2}}
\end{aligned}
$$

so

$$
\int_{0}^{\phi} d \phi^{\prime}\left(1-\alpha \cos \phi^{\prime}\right)^{2} \mathrm{e}^{\beta \cos \phi^{\prime}} \approx 2 \pi e^{\beta} H(\beta, \phi) .
$$

Comparing Eq. (A156) with Eq. (A197) we have, for $\beta \gg 1$,

$$
S_{I}(\beta, \phi) \rightarrow H(\beta, \phi) e^{\beta} .
$$

The total integral, for $\beta \gg 1$, is, from Eq. (A196)

$$
\begin{aligned}
2 \pi \mathrm{e}^{\beta} & \left(\lim _{\phi \rightarrow \infty} H(\alpha, \beta, \phi)+\lim _{\phi \rightarrow-\infty} H(\alpha, \beta, \phi)\right) \\
& \approx \mathrm{e}^{\beta} \sqrt{\frac{\pi}{2}} \frac{4 \beta^{2}(1-\alpha)^{2}}{\beta^{5 / 2}} \\
& =\mathrm{e}^{\beta} \sqrt{\frac{2 \pi}{\beta}}(1-\alpha)^{2}
\end{aligned}
$$

in agreement with Eq. (A171). Then, using Eq. (A198), Eq. (A187) becomes

$$
G_{m}(x, \phi) \cong \frac{\nu^{2 m}(x+y)^{2 m}}{m ! m} \mathrm{e}^{-\frac{q_{0} s^{2}}{4 m}} H(\beta / m, \phi)
$$

and Eq. (A193) becomes

$$
G_{0}(x, \phi) \cong \mathrm{e}^{-\frac{q_{0} \tau^{2}}{4(x+y)^{2}}} H\left(\beta^{\prime}, \phi\right)
$$

\section{d. Exponential autocorrelation: Probability distributions}

For an exponential autocorrelation function, the diffuse scattering joint probability distribution is, from Eq. (A122),

$$
\begin{aligned}
P(x, \phi)= & \frac{R}{2 N_{0} \pi y} \frac{s^{2}(1+x y-h \zeta)^{2}}{(x+y)^{2}} \\
& \times e^{-g} \sum_{m=1}^{\infty} \frac{g^{m}}{m ! m^{2}\left[1+\frac{s^{2}\left(2-x^{2}-y^{2}-2 h \zeta\right)}{m^{2}}\right]^{3 / 2}} \\
= & \frac{R}{2 N_{0} \pi y} \frac{s^{2}(1+x y)^{2}(1-\alpha \cos \phi)^{2}}{(x+y)^{2}} \mathrm{e}^{-\nu^{2}(x+y)^{2}} \\
& \times \sum_{m=1}^{\infty} \frac{\nu^{2 m}(x+y)^{2 m}}{m ! m^{2}\left[1+\frac{s^{2}\left(2-x^{2}-y^{2}\right)}{m^{2}}-\frac{4 \beta \cos \phi}{m^{2}}\right]^{3 / 2}} .
\end{aligned}
$$

\section{Marginal probability distribution in $x$}

From Eq. (A141),

$P_{x}(x)=\int_{0}^{2 \pi} d \phi P(x, \phi)=\frac{R}{2 N_{0} \pi y} \frac{\mathrm{e}^{-\nu^{2}(x+y)^{2}}}{(x+y)^{2}} \sum_{m=1}^{\infty} F_{m}(x)$

in which 


$$
\begin{aligned}
F_{m}(x)= & s^{2}(1+x y)^{2} \frac{\nu^{2 m}(x+y)^{2 m}}{m ! m^{2}} \\
& \times \int_{0}^{2 \pi} d \phi \frac{(1-\alpha \cos \phi)^{2}}{\left[1+\frac{s^{2}\left(2-x^{2}-y^{2}\right)}{m^{2}}-\frac{4 \beta \cos \phi}{m^{2}}\right]^{3 / 2}} \\
= & \frac{s^{2}(1+x y)^{2} m \nu^{2 m}(x+y)^{2 m}}{m !\left[m^{2}+s^{2}\left(2-x^{2}-y^{2}\right)\right]^{3 / 2}} \\
& \times \int_{0}^{2 \pi} d \phi \frac{(1-\alpha \cos \phi)^{2}}{\left(1-\delta_{m} \cos \phi\right)^{3 / 2}} \\
= & \frac{m \nu^{2 m}(x+y)^{2 m} \sqrt{1+x y}}{2 \sqrt{2} m ! s} \\
& \times\left(\frac{\delta_{m}}{\alpha}\right)^{3 / 2} \int_{0}^{2 \pi} d \phi \frac{(1-\alpha \cos \phi)^{2}}{\left(1-\delta_{m} \cos \phi\right)^{3 / 2}}
\end{aligned}
$$

with

$$
\begin{aligned}
\delta_{m} & =\frac{4 \beta}{m^{2}+s^{2}\left(2-x^{2}-y^{2}\right)} \\
& =\frac{2 h}{\frac{m^{2}}{s^{2}}+2-x^{2}-y^{2}} .
\end{aligned}
$$

Then we use

$$
\begin{aligned}
& \int_{0}^{2 \pi} d \phi \frac{(1-\alpha \cos \phi)^{2}}{\left(1-\delta_{m} \cos \phi\right)^{3 / 2}} \\
& =4\left[g_{1}\left(\delta_{m}\right) E\left(\frac{2 \delta_{m}}{\delta_{m}-1}\right)+g_{2}\left(\delta_{m}\right) K\left(\frac{2 \delta_{m}}{\delta_{m}-1}\right)\right]
\end{aligned}
$$

in which

$$
\begin{gathered}
g_{1}(\delta)=\frac{\delta^{2}-2 \alpha \delta-\alpha^{2}\left(\delta^{2}-2\right)}{\delta^{2}(1+\delta) \sqrt{1-\delta}} \\
g_{2}(\delta)=\frac{2 \alpha(\delta-\alpha)}{\delta^{2} \sqrt{1-\delta}}
\end{gathered}
$$

and the elliptic integrals are

$$
\begin{gathered}
E(\phi \mid \kappa)=\int_{0}^{\phi} d \theta \sqrt{1-\kappa \sin ^{2} \theta} \\
E(\kappa)=E\left(\frac{\pi}{2} \mid \kappa\right) \\
F(\phi \mid \kappa)=\int_{0}^{\phi} \frac{d \theta}{\sqrt{1-\kappa \sin ^{2} \theta}} \\
K(\kappa)=F\left(\frac{\pi}{2} \mid \kappa\right) .
\end{gathered}
$$

Thus,

$$
\begin{aligned}
F_{m}(x)= & \frac{\sqrt{2} m \nu^{2 m}(x+y)^{2 m} \sqrt{1+x y}}{m ! s}\left(\frac{\delta_{m}}{\alpha}\right)^{3 / 2} \\
& \times\left[g_{1}\left(\delta_{m}\right) E\left(\frac{2 \delta_{m}}{\delta_{m}-1}\right)+g_{2}\left(\delta_{m}\right) K\left(\frac{2 \delta_{m}}{\delta_{m}-1}\right)\right] .
\end{aligned}
$$

Large $g$ limit For $g \gg 1$, from Eq. (A137), we have

$$
\begin{aligned}
P(x, \phi)= & \frac{R}{2 N_{0} \pi y} \frac{\tau^{4}(1+x y-h \zeta)^{2}}{s^{2}(x+y)^{6}} \\
& \times \frac{1}{\left[1+\frac{\tau^{4}\left(2-x^{2}-y^{2}-2 h \zeta\right)}{(x+y)^{4} s^{2}}\right]^{3 / 2}} \\
= & \frac{R}{2 N_{0} \pi y} \frac{\tau^{4}(1+x y)^{2}(1-\alpha \cos \phi)^{2}}{s^{2}(x+y)^{6}} \\
& \times \frac{1}{\left[1+\frac{\tau^{4}\left(2-x^{2}-y^{2}\right)}{(x+y)^{4} s^{2}}-\frac{2 h \tau^{4} \cos \phi}{(x+y)^{4} s^{2}}\right]^{3 / 2}}
\end{aligned}
$$

so

$$
\begin{aligned}
P_{x}(x)= & \int_{0}^{2 \pi} d \phi P(x, \phi)=\frac{R s \tau^{4}(1+x y)^{2}}{2 N_{0} \pi y} \int_{0}^{2 \pi} d \phi \\
& \times \frac{(1-\alpha \cos \phi)^{2}}{\left[(x+y)^{4} s^{2}+\tau^{4}\left(2-x^{2}-y^{2}\right)-2 h \tau^{4} \cos \phi\right]^{3 / 2}} \\
= & \frac{R s \sqrt{1+x y}}{4 N_{0} \pi y \sqrt{2} \tau^{2}}\left(\frac{\delta_{0}}{\alpha}\right)^{3 / 2} \int_{0}^{2 \pi} d \phi \frac{(1-\alpha \cos \phi)^{2}}{\left(1-\delta_{0} \cos \phi\right)^{3 / 2}}
\end{aligned}
$$

with

$$
\delta_{0}=\frac{2 h \tau^{4}}{(x+y)^{4} s^{2}+\tau^{4}\left(2-x^{2}-y^{2}\right)} .
$$

Using

$$
\begin{aligned}
& \int_{0}^{2 \pi} d \phi \frac{(1-\alpha \cos \phi)^{2}}{\left(1-\delta_{0} \cos \phi\right)^{3 / 2}} \\
& \quad=4\left[g_{1}\left(\delta_{0}\right) E\left(\frac{2 \delta_{0}}{\delta_{0}-1}\right)+g_{2}\left(\delta_{0}\right) K\left(\frac{2 \delta_{0}}{\delta_{0}-1}\right)\right]
\end{aligned}
$$

we have

$$
\begin{aligned}
P_{x}(x)= & \frac{R s \sqrt{1+x y}}{N_{0} \pi y \sqrt{2} \tau^{2}}\left(\frac{\delta_{0}}{\alpha}\right)^{3 / 2} \\
& \times\left[g_{1}\left(\delta_{0}\right) E\left(\frac{2 \delta_{0}}{\delta_{0}-1}\right)+g_{2}\left(\delta_{0}\right) K\left(\frac{2 \delta_{0}}{\delta_{0}-1}\right)\right] .
\end{aligned}
$$




\section{Cumulative probability distribution in $\phi$}

From Eq. (A149)

$$
C_{\phi}(x, \phi)=\frac{1}{P_{x}(x)} \int_{0}^{\phi} d \phi^{\prime} P\left(x, \phi^{\prime}\right),
$$

and from Eq. (A202) and Eq. (A203), we have

$$
\int_{0}^{\phi} d \phi^{\prime} P\left(x, \phi^{\prime}\right)=\frac{R}{2 N_{0} \pi y} \frac{\mathrm{e}^{-\nu^{2}(x+y)^{2}}}{(x+y)^{2}} \sum_{m=1}^{\infty} \tilde{F}_{m}(x, \phi)
$$

in which

$$
\begin{aligned}
\tilde{F}_{m}(x, \phi)= & \frac{m \nu^{2 m}(x+y)^{2 m} \sqrt{1+x y}}{2 \sqrt{2} m ! s} \\
& \times\left(\frac{\delta_{m}}{\alpha}\right)^{3 / 2} \int_{0}^{\phi} d \phi \frac{\left(1-\alpha \cos \phi^{\prime}\right)^{2}}{\left(1-\delta_{m} \cos \phi^{\prime}\right)^{3 / 2}} .
\end{aligned}
$$

We use

$$
\begin{aligned}
\int_{0}^{\phi} & d \phi^{\prime} \frac{\left(1-\alpha \cos \phi^{\prime}\right)^{2}}{\left(1-\delta_{m} \cos \phi^{\prime}\right)^{3 / 2}} \\
= & 2 g_{0}\left(\delta_{m}, \phi\right)+2 g_{1}\left(\delta_{m}\right) E\left(\frac{\phi}{2} \mid \frac{2 \delta_{m}}{\delta_{m}-1}\right) \\
& +2 g_{2}\left(\delta_{m}\right) F\left(\frac{\phi}{2} \mid \frac{2 \delta_{m}}{\delta_{m}-1}\right)
\end{aligned}
$$

in which

$$
g_{0}(\delta, \phi)=\frac{(\alpha-\delta)^{2} \sin \phi}{\delta\left(1-\delta^{2}\right) \sqrt{1-\delta \cos \phi}} .
$$

So

$$
\begin{aligned}
\tilde{F}_{m}(x, \phi)= & \frac{m \nu^{2 m}(x+y)^{2 m} \sqrt{1+x y}}{\sqrt{2} m ! s}\left(\frac{\delta_{m}}{\alpha}\right)^{3 / 2} \\
& \times\left[g_{0}\left(\delta_{m}, \phi\right)+g_{1}\left(\delta_{m}\right) E\left(\frac{\phi}{2} \mid \frac{2 \delta_{m}}{\delta_{m}-1}\right)\right. \\
& \left.+g_{2}\left(\delta_{m}\right) F\left(\frac{\phi}{2} \mid \frac{2 \delta_{m}}{\delta_{m}-1}\right)\right]
\end{aligned}
$$

and

$C_{\phi}(x, \phi)=\frac{R}{2 P_{x}(x) N_{0} \pi y} \frac{\mathrm{e}^{-\nu^{2}(x+y)^{2}}}{(x+y)^{2}} \sum_{m=1}^{\infty} \tilde{F}_{m}(x, \phi)$.
Large g limit From Eq. (A214), we have

$$
\begin{aligned}
C_{\phi}(x, \phi)= & \frac{1}{P_{x}(x)} \int_{0}^{\phi} d \phi^{\prime} P\left(x, \phi^{\prime}\right) \\
= & \frac{R s \sqrt{1+x y}}{4 P_{x}(x) N_{0} \pi y \sqrt{2} \tau^{2}}\left(\frac{\delta_{0}}{\alpha}\right)^{3 / 2} \\
& \times \int_{0}^{\phi} d \phi^{\prime} \frac{\left(1-\alpha \cos \phi^{\prime}\right)^{2}}{\left(1-\delta_{0} \cos \phi^{\prime}\right)^{3 / 2}} \\
= & \frac{R s \sqrt{1+x y}}{2 P_{x}(x) N_{0} \pi y \sqrt{2} \tau^{2}}\left(\frac{\delta_{0}}{\alpha}\right)^{3 / 2} \\
& \times\left[g_{0}\left(\delta_{0}, \phi\right)+g_{1}\left(\delta_{0}\right) E\left(\frac{\phi}{2} \mid \frac{2 \delta_{0}}{\delta_{0}-1}\right)\right. \\
& \left.+g_{2}\left(\delta_{0}\right) F\left(\frac{\phi}{2} \mid \frac{2 \delta_{0}}{\delta_{0}-1}\right)\right] .
\end{aligned}
$$

[1] K. Ohmi, H. Jin, and Y. Susaki, Electron cloud instability in low emittance rings, in Proceedings of ECLOUD 2010: 49th ICFA Advanced Beam Dynamics Workshop on Electron Cloud Physics, edited by K. Smolenski (Cornell University, Ithaca, NY, 2013), p. 76.

[2] M. A. Furman, Electron cloud effects in accelerators, in Proceedings of ECLOUD'12, edited by R. Cimino, G. Rumolo, and F. Zimmerman (CERN, Geneva, Switzerland, 2013), p. 1.

[3] F. Zimmerman, Electron-cloud effects in past and future machines-quick walk through 50 years of electron-cloud studies, in Proceedings of ECLOUD'12, edited by R. Cimino, G. Rumolo, and F. Zimmerman (CERN, Geneva, Switzerland, 2013), p. 9.

[4] G. Iadarola and G. Rumuolo, Observations and predictions in LHC and SPS, in Proceedings of ECLOUD'12, edited by R. Cimino, G. Rumolo, and F. Zimmerman (CERN, Geneva, Switzerland, 2013), p. 19.

[5] H. Fukama, Electron cloud observations and predictions at KEKB, PEP-II and SuperB factories, in Proceedings of ECLOUD'12, edited by R. Cimino, G. Rumolo, and F. Zimmerman (CERN, Geneva, Switzerland, 2013), p. 27.

[6] G. Dugan et al., Observations and predictions at CesrTA, and outlook for ILC, in Proceedings of ECLOUD'12, edited by R. Cimino, G. Rumolo, and F. Zimmerman (CERN, Geneva, Switzerland, 2013), p. 31.

[7] G. von Holtey, A. Ball, E. Brambilla, H. Burkhardt, P. Estabrooks, F. Harris, G. Lutters, J. Rothberg, P. Roudeau, P. Siegrist, J. Wear, P. Weber, and E. Vallazza, Study of beam-induced particle backgrounds at the LEP detectors, Nucl. Instrum. Methods Phys. Res., Sect. A 403, 205 (1998).

[8] G. Dugan and D. Sagan, SYNRAD3D Photon Tracking Program, Cornell University Technical Report, 2012.

[9] M. A. Palmer et al., The conversion and operation of the Cornell electron storage ring as a test accelerator (CesrTA) for damping rings research and development, 
in Proceedings of the 23rd Particle Accelerator Conference, Vancouver, Canada, 2009 (IEEE, Piscataway, NJ, 2009), p. 4200.

[10] G. Dugan and D. Sagan, Synrad3D photon propagation and scattering simulation, in Proceedings of ECLOUD 2010: 49th ICFA Advanced Beam Dynamics Workshop on Electron Cloud Physics, edited by K. Smolenski (Cornell University, Ithaca, NY, 2013), p. 118.

[11] D. Sagan, Design and applications of the Bmad library for the simulation of particle beams and X-rays, in 11th International Computational Accelerator Physics Conference (Rostock-Warnemünde, Germany, 2012).

[12] D. Sagan, Bmad Manual, Cornell University Technical Report, 2012, http://www.lepp.cornell.edu/ dcs16/bmad/.

[13] P. Beckmann and A. Spizzichino, The Scattering of Electromagnetic Waves from Rough Surfaces (Pergamon Press, New York, 1963).

[14] J. A. Ogilvy, Theory of Wave Scattering from Random Rough Surfaces (Hilger, Bristol, 1993).

[15] B. L. Henke, E. M. Gullikson, and J. C. Davis, X-ray interactions: photoabsorption, scattering, transmission, and reflection at $E=50-30,000 \mathrm{eV}, Z=1-92$, At. Data Nucl. Data Tables 54, 181 (1993), http://henke.lbl .gov/optical_constants/layer2.html.

[16] H. Hogrefe and C. Kunz, Soft $x$-ray scattering from rough surfaces: experimental and theoretical analysis, Appl. Opt. 26, 2851 (1987).

[17] R. J. Noll and P. Glenn, Mirror surface autocovariance functions and their associated visible scattering, Appl. Opt. 21, 1824 (1982).

[18] N. Mahne, A. Giglia, S. Nannarone, R. Cimino, and C. Vaccarezza, Experimental determination of e-cloud simulation input parameters for DAFNE, in Proceedings of the 21st Particle Accelerator Conference, Knoxville, TN, 2005 (IEEE, Piscataway, NJ, 2005), p. 817.

[19] G. Dugan and D. Sagan, Synrad3D photon propagation and scattering simulations, in Proceedings of ECLOUD'12, edited by R. Cimino, G. Rumolo, and F. Zimmerman (CERN, Geneva, Switzerland, 2013), p. 117.

[20] G. Dugan, K. G. Sonnad, R. Cimino, T. Ishibashi, and F. Schafers, Measurements of x-ray scattering from accelerator vacuum chamber surfaces, and comparison with an analytical model, Phys. Rev. ST Accel. Beams 18, 040704 (2015).

[21] J. A. Crittenden et al., Investigation into electron cloud effects in the ILC damping ring design, in Proceedings of the 3rd International Particle Accelerator Conference, New Orleans, LA, 2012 (IEEE, Piscataway, NJ, 2012), p. 1963.

[22] J. A. Crittenden and J. P. Sikora, Electron cloud buildup characterization using shielded pickup measurements and custom modeling code at CESRTA, in Proceedings of ECLOUD'12, edited by R. Cimino, G. Rumolo, and F. Zimmerman (CERN, Geneva, Switzerland, 2013), p. 241.

[23] L. Boon and K. Harkay, Chamber surface roughness and electron cloud for the APS SCU, in Proceedings of ECLOUD'12, edited by R. Cimino, G. Rumolo, and F. Zimmerman (CERN, Geneva, Switzerland, 2013), p. 95.

[24] J. D. Jackson, Classical Electromagnetism, 3rd ed. (Wiley, New York, 1999).

[25] Abramowitz and Stegun, Handbook of Mathematical Functions (Dover Publications Inc., New York, 1972), p. 377. 